\title{
How Completeness and Correspondence Theory \\ Got Married
}

\author{
Marcus Kracht \\ Faculteit der Wijsbegeerte \\ Rijksuniversiteit Utrecht \\ Heidelberglaan 8 \\ 3584 CS Utrecht
}

\section{Introduction}

It has been said that modal logic consists of three main disciplines: duality theory, completeness theory and correspondence theory; and that they are the pillars on which this edifice called modal logic rests. This seems to be true if one looks at the history of modal logic, for all three disciplines have been explicitly defined around the same time, namely in the mid-seventies. While it is certainly true that modal logic can be divided in this way, such a division creates the danger that the subareas are developed merely in their own right, disregarding the obvious connections between them. Moreover, such historically grown divisions always run a risk of enshrining certain errors that have accidentally been made and subsequently hindered the development. One such error is the idea that Kripkeframes are the natural or fundamental semantics for modal logic. Although I agree that Kripke-models are the most intuitive models and that they are in many cases indeed the intended models, I cannot go along with the claim that they are in any stronger sense fundamental. Philosophically as well as mathematically, to start with Kripke-frames is to start at the wrong end; philosophically, because nothing warrants the belief that possible worlds exist - in fact, for my ears this is a contradictio in adiectu — and indeed it is much more plausible to say that possible worlds are philosophical fiction. And mathematically, because Kripke-frames are deficient in some respects and these deficiencies do not apply to modal algebras and also because the former can be obtained canonically from the latter. It is surprising how long it was possible to ignore Stone's representation theory for boolean algebras and also Tarski's work together with Jónsson in which Kripke-semantics appeared long before Kripke himself came to discover it.

One thing that could be held against algebraic semantics in general is that it is hard to understand intuitively. Plus, a philosopher might disagree with it because we have now 
committed ourselves to the non-existence of possible worlds or-to be precise- to the view that a possible world can be reconstructed as a maximal consistent set of situations. But it was already discovered in [Jónsson and Tarski, 1951] that every modal algebra A can be realized in the sense that there exists a mono-morphism $\mathbf{A} \longmapsto 2^{g}$ into the complete algebra of sets over a Kripke-frame $g$. This led to the notion of a generalized frame. Generalized frame integrates the two semantics in the sense that they are technically like modal algebras but we nevertheless always have a Kripke-frame at hand. The simplest way to think of generalized frames is as a pair $\langle g, \mathbb{G}\rangle$ where $g$ is a Kripke-frame and $\mathbb{G} \subseteq 2^{g}$ a set of sets closed under some operations. An element of $\mathbb{G}$ is called an internal set or situation because they are the sets that naturally exist for an inhabitant of this frame. Generalized frames allow for the possibility that a world cannot be uniquely constructed from situations; thus, neither does the algebra of internal sets define the Kripke-frame nor does the Kripke-frame define the algebra of internal sets up to isomorphism and thus we can study the relative interdefinability of worlds and situations without any prejudice. The pricipal aim of duality theory is to study the interconnection between Kripke-frames and modal algebras in general frames. It is this theory that is presupposed by and therefore supports both completeness and correspondence theory.

The proposal of this essay is to regard generalized frames as the fundamental structures of modal logic and to revise our terminology accordingly. In particular, we will relativize the notions of completeness and elementarity alias correspondence to classes of generalized frames. The effect of this is quite drastic. It is as easy to show that $\mathbf{K} 4=\mathbf{K}(\diamond \diamond p \rightarrow \diamond p)$ is elementary in the class of refined frames as it is to show that it is elementary in the class of Kripke-frames. The additional benefit is that we get completeness with respect to Kripke-frames for free. However, it might seem that this generalization of elementarity to elementarity-in-a-class is much too general to allow for significant results. That this is not the case is another point we want to make. We will demonstrate it with a number of examples from the literature which we will recast in this new terminology. The second half of this essay is devoted to the development of a proof calculus which derives pairs of corresponding modal formulae and elementary formulae. This calculus consists of a number of axioms and rules which are valid no matter what class we choose. In addition, the narrower the class the more axioms and rules can be added; in particular, we will show that in the class consisting of the Kripke-frames and the descriptive frames there is an additional axiom and one more rule available and they suffice to generate all formulae of Sahlqvist's Theorem. It is worthwile mastering the calculus that we will describe, which works with sequences of propositions rather than single 
propositions. Although it may look awful in the beginning, especially in the interpretation via multi-frames that we have chosen, the conceptual advantage to control several worlds at the same time will soon become apparent. An extra of the calculus is that we can also describe the elementary properties that correspond to axioms of Sahlqvist type. As it turns out, they can be described much easier than their modal counterparts. This, if anything, I hope to be a lasting contribution of this paper as it greatly simplifies the application of Sahlqvist's Theorem.

I have held for a long time the conviction that completeness and correspondence proofs both draw heavily on definability theory in the general sense and have made this the title of my dissertation [Kracht, 1991]. But although I may have succeeded in reducing completeness proofs of the most diverse kind to definability problems, the general theory of internal definitions remained a weak part of that work. Thus, when I was asked to contribute to this book I decided to sit down and write this part afresh, allowing me to incorporate new insights and much of the ideas that have been suggested to me by Kit Fine, Wolfgang Rautenberg and most prominently by Johan van Benthem. As I am now standing on their shoulders waving my flag I wish to dedicate this essay to them.

\section{The Languages of Modal Logic}

The internal language $\mathcal{L}^{i}$ of modal logic consists of a denumerable set $\mathbb{P}_{\omega}:=\left\{p_{i}: i \in \omega\right\}$ of propositional variables out of which propositions are built using the classical logical junctors $\top, \perp, \neg, \wedge, \vee, \rightarrow, \ldots$ plus the modal operators $\square, \diamond$. Lower case Roman letters such as $p, q, r, \ldots$ denote variables and upper case Roman letters $P, Q, R, \ldots$ denote propositions. As usual, a normal modal logic $\Lambda$ is a subset of $\mathcal{L}^{i}$ which contains the classical tautologies, the axiom $\square(p \rightarrow q) \rightarrow . \square p \rightarrow \square q$ and which is closed under substitution, Modus Ponens and MN: $p / \square p$. The typical models for $\mathcal{L}^{i}$ are based on modal algebras. A modal algebra is a pair $\langle\mathbf{A}, \mathbf{\square}\rangle$ where $\mathbf{A}=\langle A, 1, \cap,-\rangle$ is a boolean algebra and $\mathbf{\square}$ a operator satisfying $\square 1=1$ and $\boldsymbol{\square}(a \cap b)=\boldsymbol{\square} a \cap \boldsymbol{\square}$. A valuation is a map $\gamma: \mathbb{P} \rightarrow A$ from a subset $\mathbb{P}$ of $\mathbb{P}_{\omega}$ into the carrier set of $\mathbf{A}$. $\gamma$ can be lifted to a homomorphism $\bar{\gamma}: \mathcal{L}^{i}(\mathbb{P}) \rightarrow \mathbf{A}$ in the natural way. For reasons to become clear we call an ultrafilter of $\mathbf{A}$ a point. Given $\mathbf{A}$, a valuation $\gamma$ and a point $\mathfrak{U}$ we write $\langle\mathbf{A}, \gamma, \mathfrak{U}\rangle \vDash P$ for a formula based on variables from $\operatorname{dom}(\gamma)$ if $\bar{\gamma}(P) \in \mathfrak{U}$. By abstraction we write $\langle\mathbf{A}, \gamma\rangle \vDash P$ if for all $\mathfrak{U}\langle\mathbf{A}, \gamma, \mathfrak{U}\rangle \vDash P$; equivalently, $\langle\mathbf{A}, \gamma\rangle \vDash P$ if $\bar{\gamma}(P)=1$. Likewise $\mathbf{A} \vDash P$ or $\langle\mathbf{A}, \mathfrak{U}\rangle \vDash P$ are used; the nonoc- 
curring symbols are implicitly universally quantified. We write $\operatorname{Th}(\mathbf{A})=\{P: \mathbf{A} \vDash P\}$ and $\operatorname{Md}(\Lambda)=\{\mathbf{A}: \mathbf{A} \vDash \Lambda\}$.

Just as the internal language is directly connected with the algebraic semantics in the sense that each of the symbols has a direct counterpart in the algebras, so is the language of frames $\mathcal{L}^{f}$ connected with geometrical models or Kripke-frames. $\mathcal{L}^{f}$ has a denumerable set $\mathbb{W}_{\omega}:=\left\{w_{i}: i \in \omega\right\}$ of world-variables which will be denoted by the letters $v, w, x, \ldots$ Formulas are built using the relational symbols of equality $(\dot{=})$ and accessibility $(\triangleleft)$ and the logical connectives true, false, $\sim, \&, \vee, \ldots$ with the quantifiers $\forall, \exists . \alpha \in \mathcal{L}^{f}$ is called an elementary formula. The first-order models of $\mathcal{L}^{f}$ are pairs $g=\langle g, \triangleleft\rangle$ with $\triangleleft \subseteq g^{2}$. In this context these models are called Kripke-frames. A valuation is a function $\iota: \mathbb{W} \rightarrow g$.

The external language $\mathcal{L}^{e}$ of modal logic is a fragment of second-order monadic predicate logic as in [Benthem, 1983]. Conceptually as well as mathematically, we see some advantages in construing it here as a fragment of two-sorted, first-order predicate logic. One sort is then the sort of propositions and the other the sort of worlds. $\mathcal{L}^{e}$ fuses both $\mathcal{L}^{i}$ and $\mathcal{L}^{f}$; it contains all symbols of these languages. In addition it has quantifiers over propositions and a binary symbol $\epsilon$ denoting acceptance. Given a world $w$ and a proposition $P w \in P$ means that $w$ accepts $P$ or that $w$ is in the denotation of $P$ etc. It is tempting to conclude that the natural models for $\mathcal{L}^{e}$ are generalized frames, which consist of a Kripke-frame $g$ together with a subset $\mathbb{G} \subset 2^{g}$ closed under intersection, comlementation and $\square a:=\{s:(\forall t)(s \triangleleft t \Rightarrow t \in a)\}$ (and we can implicitly see it as an algebra). But there is a snag; for $\mathcal{L}^{e}$ exceeds $\mathcal{L}^{i}$ and $\mathcal{L}^{f}$ by having quantifiers for propositions. However, for the definition of the satisfaction of a formula we need not worry about such details although this point will be of some significance later on. Now, given a generalized frame $\mathcal{G}=\langle g, \mathbb{G}\rangle$ and two maps $\gamma: \mathbb{P} \rightarrow A, \iota: \mathbb{W} \rightarrow g$ we define $\langle\mathcal{G}, \gamma, \iota\rangle \vDash \alpha$ for $\alpha$ with free variables in $\operatorname{dom}(\gamma)$ vz. $\operatorname{dom}(\iota)$ by induction. The base clauses are as follows.

$$
\begin{array}{llll}
\langle\mathcal{G}, \gamma, \iota\rangle & \models v \doteq w & \text { iff } & \iota(v)=\iota(w) \\
\langle\mathcal{G}, \gamma, \iota\rangle \vDash v \triangleleft w & \text { iff } & \iota(v) \triangleleft \iota(w) \\
\langle\mathcal{G}, \gamma, \iota\rangle \vDash v \in P & \text { iff } & \iota(v) \in \bar{\gamma}(P)
\end{array}
$$

It is worthwile to introduce some abbreviations. Let us write $v \triangleleft^{0} w$ if $v \doteq w$ and $v \triangleleft^{k+1} w$ for $(\exists x)\left(v \triangleleft x \triangleleft^{k} w\right)$. For a finite set $\kappa$ of natural numbers let $v \triangleleft^{\kappa} w:=\bigvee\left\langle v \triangleleft^{\ell} w\right.$ : $\ell \in \kappa\rangle$. We will also use restricted quantifiers of type $\forall w \triangleright^{\kappa} v, \exists w \triangleright^{\kappa} v$ which will have a straightforward translation: 


$$
\begin{aligned}
& \left(\exists w \triangleright^{\kappa} v\right) \alpha \leftrightharpoons(\exists w)\left(v \triangleleft^{\kappa} w \cdot \& . \alpha\right) \\
& \left(\forall w \triangleright^{\kappa} v\right) \alpha \leftrightharpoons(\forall w)\left(v \triangleleft^{\kappa} w . \supset . \alpha\right)
\end{aligned}
$$

The variable $v$ is called the restrictor of the quantifiers $\forall w \triangleright^{k} v, \exists w \triangleright^{k} v$. Although the quantifiers $\forall w \triangleleft^{\kappa} v, \exists w \triangleright^{\kappa} v$ are in fact multiple quantifiers in their translation (since they are hiding variables), the quantificational force of them is pure in the sense that $\forall w \triangleright^{\kappa} v$ is a universal quantifier for all hidden variable and likewise $\exists w \triangleright^{k} v$ quantifies existentially all variables in its translation. In the special case of the simple restiucted existentials and universals note that if $f r(\alpha)$ is the set of free variables of $\alpha$ then we must have $f r((\exists y \triangleright x) \beta)=f r((\forall y \triangleright x) \beta)=\{x\} \cup f r(\beta)$. The typical laws for quantifiers hold also for their restricted counterparts e.g. $\sim\left(\forall w \triangleright^{k} v\right) \alpha \equiv\left(\exists w \triangleright^{k} v\right) \sim \alpha$. Note that $\left(\forall w \triangleright^{0} v\right) \alpha \equiv \alpha[w / v]$. A formula $\alpha$ is called restricted if it is built with the help of restricted quantifiers only with the exception of the outermost quantifier in case $\alpha$ is a sentence. If $\alpha$ is built from positive or negative atoms using only $\wedge, \vee, \forall$ and restricted existential quantifiers, $\alpha$ is called existentially restricted.

We call the triple $\langle\mathcal{G}, \gamma, \iota\rangle$ an e-model. Given two generalized frames $\langle g, \mathbb{G}\rangle,\langle h, \mathbb{H}\rangle$ and a set map $p: g \rightarrow h$ (not necessarily a p-morphism) then $p$ naturally induces a map $p^{+}: 2^{h} \rightarrow 2^{g}: a \mapsto p^{-1}[a]$. If $p^{+}[\mathcal{H}] \subseteq \mathcal{G}$ and we have an e-model $\langle\mathcal{G}, \gamma, \iota\rangle$ based on $\mathcal{G}$ then $p$ also induces a map between e-models $p:\langle\mathcal{G}, \gamma, \iota\rangle \rightarrow\langle\mathcal{G}, \delta, \kappa\rangle$ with $\kappa=p \circ \iota$ and $\gamma=p^{+} \circ \delta$.

\section{Classes of Generalized Frames}

Given a generalized frame $\mathcal{G}=\langle g, \mathbb{G}\rangle$ and an arbitrary set $a \subseteq g$ we call $a$ internal if $a \in \mathbb{G} . \mathcal{G}$ defines a Kripke-frame $\mathcal{G}_{\sharp}:=\langle g, \triangleleft\rangle$ and a modal algebra $\mathcal{G}_{+}:=\langle\mathbb{G}, \cap,-, \mathbf{\square}\rangle$ in a straightforward way. Every world $w$ of $\mathcal{G}$ also uniquely determines the point $\mathfrak{U}_{w}=\{a$ : $w \in a\}$. Also, given a Kripke-frame $g$ this frame can naturally be viewed as a generalized frame via the identification $g^{\sharp}:=\left\langle g, 2^{g}\right\rangle$. Given a class $\mathfrak{X}$ of generalized frames we denote by $\mathfrak{X}^{\bullet}$ the class $\mathfrak{X} \cup\left\{\left(\mathcal{G}_{\sharp}\right)^{\sharp}: \mathcal{G} \in \mathfrak{X}\right\}$. If $\mathfrak{X}=\mathfrak{X}^{\bullet}$ let us agree to call $\mathfrak{X}$ closed. Finally, given a modal algebra $\mathbf{A}$ we can use Stone-representation to get a generalized frame $\mathbf{A}^{+}$. The points of $\mathbf{A}^{+}$are the points (= ultrafilters) of $\mathbf{A}$ and $\mathfrak{U} \triangleleft \mathfrak{T} \Leftrightarrow \triangleleft \mathfrak{T} \subseteq \mathfrak{U}$ where $\checkmark \mathfrak{T}=\{a: a \in \mathfrak{T}\}$. Finally, the internal sets are the sets $\widehat{a}:=\{\mathfrak{U}: a \in \mathfrak{U}\}$. The maps $-_{\sharp}$ and $-^{\sharp}$ are covariant functors between the category Gfr of generalized frames and the category Frm of Kripke-frames; the maps $--_{+}$and $-^{+}$are contraviant functors 
between the category Mal of modal algebras and Gfr. The arrows in these categories are homomorphisms in Mal, p-morphisms in Frm and Gfr. (Recall that a map $p: \mathcal{G} \rightarrow \mathcal{H}$ must define a map $p_{\sharp}: \mathcal{G}_{\sharp} \rightarrow \mathcal{H}_{\sharp}$ between the underlying Kripke-frames. Given $p_{\sharp}$, there is a unique map $p^{+}: \mathcal{H}^{+} \rightarrow \mathcal{G}^{+}$defined by $p^{+}(a)=p_{\sharp}^{-1}[a]$. So a p-morphism between generalized frames can safely be confused with the p-morphism between the underlying Kripke-frames, a policy which we will adopt here. More one the subject of duality theory can be found in [Sambin and Vaccaro, 1988]. Note that in our notation the forgetful functor is always written as a subscript, the recovery functor as a superscript.) By convention we write $\rightarrow$ for an arrow in a category; we write $\rightarrow$ for surjective and $\longmapsto$ for injective morphisms. By $\rightarrow$ we denote a map which is not a morphism. We say that $\mathcal{G}$ is $n$-generated (finitely generated) if the underlying algebra $\mathcal{G}_{+}$is $n$-generated (finitely generated). In a similar vein we attribute algebraic properties to $\mathcal{G}$ if $\mathcal{G}_{+}$has these properties and we attribute frame properties to $\mathcal{G}$ whenever the underlying frame $\mathcal{G}_{\sharp}$ has them.

From now on let us agree to call generalized frames simply frames, which is normally reserved for Kripke-frames; the latter will therefore be referred to consistently as Kripkeframes. Generalized frames are categorized into various classes. $\mathcal{G}$ is differentiated if for all $s, t s=t \Leftrightarrow(\forall a \in \mathbb{G})(s \in a \Leftrightarrow t \in a)$. $\mathcal{G}$ is tight if for all $s, t s \triangleleft t \Leftrightarrow(\forall a \in$ $\mathbb{G})(t \in a \Rightarrow s \in a)$. $\mathcal{G}$ is refined if $\mathcal{G}$ is both differentiated and tight. (Note that all three notions can be expressed by a universal $\mathcal{L}^{e}$ sentence.) We call $\mathcal{G}$ full if $\mathcal{G} \cong\left(\mathcal{G}_{\sharp}\right)^{\sharp}$; in other words $\mathcal{G}$ is full if it is a Kripke-frame modulo identification of $g$ with $g^{\sharp}$. Finally, $\mathcal{G}$ is said to be descriptive if $\mathcal{G} \cong\left(\mathcal{G}_{+}\right)^{+}$; this is equivalent with $\mathcal{G}$ being refined and compact as a topological space. Another characterization for descriptive frames is that they are refined and for every ultrafilter $\mathfrak{U} \cap \mathfrak{U} \neq \varnothing$ ([Goldblatt, 1976]). These classes of frames are denoted by the following symbols: $\mathfrak{G}$ for the class of (generalized) frames, $\mathfrak{D} \mathfrak{f}$ for the differentiated, $\mathfrak{T} \mathfrak{i}$ for the tight, $\mathfrak{R}$ for the refined frames; $\mathfrak{D}$ for descriptive, $\mathfrak{K}$ for Kripke-frames (alias full frames); $\mathfrak{F}$ for finite Kripke-frames.

There is a number of classes that will play an important role in this paper though they are mostly of marginal interest. First we have the class of frames in which Esakia's Lemma holds. Recall that Esakia's Lemma states that for every upward directed family $\Delta$ of internal sets of a descriptive frame $\mathcal{G} \square \lim \Delta=\lim \square \Delta$. For downward directed families this trivially holds in all frames. Here, an upward directed family is a family $\left\langle d_{p}: p \in P\right\rangle$ of sets indexed by a poset $\langle P, \leq\rangle$ such that (i) $p \leq q \Rightarrow d_{p} \subseteq d_{q}$ and (ii) for all $p, q \in P$ there is an $r \in P$ such that $p, q \leq r$. Then $\lim \Delta$ is simply the union $\bigcup\left\langle d_{p}: p \in P\right\rangle$ 
whereas for a downward going family $\Delta$ the limit is $\bigcap\left\langle d_{p}: p \in P\right\rangle$. Let us call a frame continuous or an Esakia-frame if it satisfies this property and let us abbreviate the class of Esakia-frames by $\mathfrak{E}$. Esakia's Lemma now has the form $\mathfrak{D} \subseteq \mathfrak{E}$. By induction, if $P$ is formula free of $\neg$ and $\Delta$ an up- or downward family then $P[\Delta]$ is an up- or downward family and $\lim P[\Delta]=P[\lim \Delta]$.

Next recall from [Fine, 1975] the notion of a modally saturated frame. $\mathcal{G}$ is modally 1-saturated if for every point $\mathfrak{U} \varnothing \neq \cap \mathfrak{U}$ and $\mathcal{G}$ is modally 2-saturated if for every pair $\mathfrak{T}, \mathfrak{U}$ such that $\triangleleft \mathfrak{U} \subseteq \mathfrak{T}$ then for each $t \in \cap \mathfrak{T}$ there is a $u \in \cap \mathfrak{U}$ with $t \triangleleft u$. Finally, $\mathcal{G}$ is modally saturated if it is both modally 1- and 2-saturated. [Goldblatt, 1976] calls such frames replete. Note that if a frame $\mathcal{G}$ is 2 -saturated then the 1-refinement map which identifies two worlds $t, u$ if $\mathfrak{U}_{t}=\mathfrak{U}_{u}$ is a p-morphism onto a refined frame. It follows that the refinement of a saturated frame is a descriptive frame. If we speak of the class of frames which have a $p$-morphic image in $\mathfrak{X}$ as rough $\mathfrak{X}$-frames we can say that the class $\mathfrak{S a t}$ of saturated frames is the class of rough $\mathfrak{D}$-frames. It follows that $\mathfrak{S} \mathfrak{a t} \subseteq \mathfrak{E}$. Finally, we define an approximation frame to be a frame which is either continuous or a rough $\mathfrak{K}$-frame. In this context it is only of interest that rough Kripke-frames have complete intersection and union with full distributivity. The class of approximation frames is defined by $\mathfrak{A}$.

There is an interesting construction due to [Fine, 1975] with which to turn a frame $\langle g, \mathbb{G}\rangle$ into a saturated frame $\langle h, \mathbb{H}\rangle$ such that $g$ can be elementarily embedded into $h$ and the restriction map $a \mapsto a \cap g$ defines an isomorphism between $\langle\mathbb{H}, 1, \cap,-, \mathbf{\square}\rangle$ and $\langle\mathbb{G}, 1, \cap,-, \mathbf{\square}\rangle$. Simply adjoin to $\mathcal{L}^{f}$ a unary predicate $\underline{a}$ for each $a \in \mathbb{G}$; this defines $\mathcal{L}^{f}(\mathbb{G})$. Now let $g \preccurlyeq h$ be a $\omega$-saturated elementary extension (for example some ultrapower of $g$ ) in $\mathcal{L}^{f}(\mathbb{G})$ and put $\llbracket \underline{a} \rrbracket=\{x \in h: \underline{a}(x)\}$. By grinding out the details one can prove that $a \mapsto \llbracket \underline{a} \rrbracket$ is an isomorphism inverse to the restriction map and that $\langle h, \mathbb{H}\rangle$ is modally saturated. $\langle h, \mathbb{H}\rangle$ is not necessarily refined since there are far more $\mathcal{L}^{f}(\mathbb{G})$-types as there are modal types.

\section{Completeness, Persistence and the Like}

Writing the shorthand $\operatorname{Md}_{\mathfrak{X}}(\Lambda)=\operatorname{Md}(\Lambda) \cap \mathfrak{X}$ we can state the following definition.

Definition 1 Let $\mathfrak{X}$ be a class of generalized frames and $\Lambda$ be a logic. We say that $\Lambda$ is 
$\mathfrak{X}$-complete if $\Lambda=\operatorname{Th}\left(\operatorname{Md}_{\mathfrak{X}}(\Lambda)\right.$ ); that $\Lambda$ is $\mathfrak{X}$-persistent if $\operatorname{Md}_{\mathfrak{X}}(\Lambda)^{\bullet}=\operatorname{Md}_{\mathfrak{X}} \cdot(\Lambda)$. Finally, $\Lambda$ is $\mathfrak{X}$-elementary if there is an elementary sentence $\alpha$ such that $\operatorname{Md}_{\mathfrak{X}}(\Lambda)=\operatorname{Md}_{\mathfrak{X}}(\alpha)$, $\mathfrak{X}-\Delta$-elementary if there is a set $\Gamma$ of elementary sentences such that $\operatorname{Md}_{\mathfrak{X}}(\Lambda)=\operatorname{Md}_{\mathfrak{X}}(\Gamma)$; and $\Lambda$ is $\mathfrak{X}-\Sigma \Delta$-elementary if $\operatorname{Md}_{\mathfrak{X}}(\Lambda)$ is a union of $\mathfrak{X}-\Delta$-elementary classes.

These definitions are somewhat compact versions of the usual definitions. $\Lambda$ is complete if $P$ is a theorem of $\Lambda$ exactly if it holds in all Kripke-frames contained in $\mathfrak{X} ; \Lambda$ is $\mathfrak{X}$ persistent if for all $\mathfrak{X}$-frames $\mathcal{G}$ we have $\left(\mathcal{G}_{\sharp}\right)^{\sharp} \vDash \Lambda$ if and only if $\mathcal{G} \vDash \Lambda$. Note that we do not require that $\left(\mathcal{G}_{\sharp}\right)^{\sharp} \in \mathfrak{X} . \Lambda$ is $\mathfrak{X}$-elementary if it selects an elementary class of frames from $\mathfrak{X}$. The following facts are easy consequences of the definitions.

Fact 2 Suppose that $\mathfrak{X} \subseteq \mathfrak{Y}$. Then if $\Lambda$ is $\mathfrak{X}$-complete it is also $\mathfrak{Y}$-complete.

Proof. If $\mathfrak{X} \subseteq \mathfrak{Y}$ then $\operatorname{Md}_{\mathfrak{X}}(\Lambda) \subseteq \operatorname{Md}_{\mathfrak{Y}}(\Lambda)$ whence $\operatorname{Th}\left(\operatorname{Md}_{\mathfrak{X}}(\Lambda)\right) \supseteq \operatorname{Th}\left(\operatorname{Md}_{\mathfrak{Y}}(\Lambda)\right)$. Since $\Lambda \subseteq \operatorname{Th}\left(\operatorname{Md}_{\mathfrak{Y}}(\Lambda)\right)$ and by assumption $\Lambda=\operatorname{Th}\left(\operatorname{Md}_{\mathfrak{X}}(\Lambda)\right)$, it follows that $\Lambda=\operatorname{Th}\left(\operatorname{Md}_{\mathfrak{Y}}(\Lambda)\right)$.

Fact 3 Suppose that $\left\langle\Lambda_{i}: i \in I\right\rangle$ is a family of $\mathfrak{X}$-complete logics. Then $\bigcap\left\langle\Lambda_{i}: i \in I\right\rangle$ is $\mathfrak{X}$-complete as well.

Proof. $\operatorname{Th}\left(\operatorname{Md}_{\mathfrak{X}}\left(\bigcap\left\langle\Lambda_{i}: i \in I\right\rangle\right)\right)=\operatorname{Th}\left(\bigcup\left\langle\operatorname{Md}_{\mathfrak{X}}\left(\Lambda_{i}\right): i \in I\right\rangle\right)=\bigcap\left\langle\operatorname{Th}\left(\operatorname{Md}_{\mathfrak{X}}\left(\Lambda_{i}\right)\right): i \in I\right\rangle=$ $\bigcap\left\langle\Lambda_{i}: i \in I\right\rangle$.

Fact 4 Let $\mathfrak{X} \supseteq \mathfrak{Y}$ and $\Lambda$ be $\mathfrak{X}$-persistent ( $\mathfrak{X}$-elementary). Then $\Lambda$ is also $\mathfrak{Y}$-persistent (Y)-elementary).

Proof. If $\mathfrak{X} \supseteq \mathfrak{Y}$ then $\mathfrak{X}^{\bullet} \supseteq \mathfrak{Y}^{\bullet}$ and so $\operatorname{Md}_{\mathfrak{Y}}(\Lambda)^{\bullet}=\operatorname{Md}_{\mathfrak{X}}(\Lambda)^{\bullet} \cap \mathfrak{Y}^{\bullet}=\operatorname{Md}_{\mathfrak{X}^{\bullet}}(\Lambda) \cap \mathfrak{Y}^{\bullet}=$ $\operatorname{Md}_{\mathfrak{Y}} \cdot(\Lambda)$.

Fact 5 Let $\left\langle\Lambda_{i}: i \in I\right\rangle$ be a family of $\mathfrak{X}$-persistent (X-elementary) logics. Then $\bigsqcup\left\langle\Lambda_{i}\right.$ : $i \in I\rangle$ is $\mathfrak{X}$-persistent ( $\mathfrak{X}-\Delta$-elementary).

Proof. The claim concerning elementarity is trivial. $\operatorname{Md}_{\mathfrak{X}}\left(\bigsqcup\left\langle\Lambda_{i}: i \in I\right\rangle\right)^{\bullet}=\bigcap\left\langle\operatorname{Md}_{\mathfrak{X}}\left(\Lambda_{i}\right)\right.$ : $i \in I\rangle^{\bullet}=\bigcap\left\langle\operatorname{Md}_{\mathfrak{X}}\left(\Lambda_{i}\right)^{\bullet}: i \in I\right\rangle=\bigcap\left\langle\operatorname{Md}_{\mathfrak{x}}\left(\Lambda_{i}\right): i \in I\right\rangle=\operatorname{Md}_{\mathfrak{X}}\left(\bigsqcup\left\langle\Lambda_{i}: i \in I\right\rangle\right)$.

The connection between these properties is highlighted by some easy observations. 
Proposition 6 If $\Lambda$ is $\mathfrak{X}$-persistent and $\mathfrak{X}$-complete, then $\Lambda$ is also $\mathfrak{X}^{\bullet} \cap \mathfrak{K}$-complete.

Proof. Let $\mathfrak{C}$ a class of frames. We not only have $\operatorname{Th}\left(\mathfrak{C}^{\bullet}\right) \subseteq \operatorname{Th}(\mathfrak{C})$ but in fact $\operatorname{Th}\left(\mathfrak{C}^{\bullet} \cap \mathfrak{K}\right) \subseteq$ $\mathrm{Th}(\mathfrak{C})$ since for every frame the logic of the underlying Kripke-frame is weaker than the logic of the frame itself. Thus $\operatorname{Th}\left(\operatorname{Md}_{\mathfrak{X} \bullet \cap \mathfrak{K}}(\Lambda)\right) \subseteq \operatorname{Th}\left(\operatorname{Md}_{\mathfrak{X}}(\Lambda)\right)$ since $\operatorname{Md}_{\mathfrak{X} \bullet \cap \mathfrak{K}}(\Lambda)^{\bullet}=$ $\operatorname{Md}_{\mathfrak{X}}(\Lambda)^{\bullet} \cap \mathfrak{K}$. Now $\Lambda \subseteq \operatorname{Th}\left(\operatorname{Md}_{\mathfrak{X} \bullet \cap \mathfrak{K}}(\Lambda)\right) \subseteq \operatorname{Th}\left(\operatorname{Md}_{\mathfrak{X}}(\Lambda)\right)=\Lambda$ and $\operatorname{so} \Lambda=\operatorname{Th}\left(\operatorname{Md}_{\mathfrak{X} \bullet \cap \mathfrak{K}}(\Lambda)\right)$.

Proposition 7 If $\Lambda$ is $\mathfrak{X}^{\bullet}$-elementary then $\Lambda$ is also $\mathfrak{X}^{\bullet}$-persistent.

Proof. It is enough to show this for $\Delta$-elementary classes. Here we have $\operatorname{Md}_{\mathfrak{X}}(\Lambda)^{\bullet}=$ $\operatorname{Md}_{\mathfrak{X}^{\bullet}}(\Gamma)^{\bullet}=\operatorname{Md}_{\mathfrak{X}^{\bullet}}(\Gamma)=\operatorname{Md}_{\mathfrak{X}^{\bullet}}(\Lambda)$ simply because a generalized frame satisfies $\Gamma$ iff the underlying Kripke-frame satisfies $\Gamma$.

Suppose that $\mathfrak{X}^{\bullet}$ is 'big' in the sense that every logic is $\mathfrak{X}^{\bullet}$-complete. Then if $\Lambda$ is $\mathfrak{X}^{\bullet}$ elementary, $\Lambda$ is also $\mathfrak{X}^{\bullet} \cap \mathfrak{K}$-complete and hence Kripke-complete. So in order to prove completeness with respect to some class of Kripke-frames one can prove persistence with respect to some suitable class of generalized frames and to show persistence it suffices to prove elementarity in that class. However, as the above theorem tells us, completeness with respect to Kripke-frames is conditional on a completeness proof with respect to the same class one has proved persistence for. For when $\mathfrak{X}$ is too small, for example when $\mathfrak{X}=\mathfrak{K}$ then this argument is otherwise invalid. One may wonder whether elementarity, completeness and persistence always go hand in hand like this. But there are counterexamples. For instance, McKinsey's logic has the finite model property but does not define an elementary class of finite frames. It is also not canonical (see [Goldblatt, 1991]). However, the following was noted in [Fine, 1975], of which it is unknown whether the converse holds.

Theorem 8 Let $\mathfrak{X} \subseteq \mathfrak{K}$ be a $\Delta$-elementary class. If $\Lambda$ is $\mathfrak{X}$-complete and $\mathfrak{X}$ - $\Sigma \Delta$-elementary then $\Lambda$ is $\mathfrak{D}$-persistent.

Proof. Let $\mathcal{F}_{\Lambda}(k)$ be the freely $k$-generated $\Lambda$-algebra. If $\mathbb{P}=\left\{p_{i}: i<k\right\}$ and $\operatorname{var}(P) \subseteq \mathbb{P}$ then if $P$ is $\Lambda$-consistent there is a model $\left\langle g_{P}, \gamma_{P}\right\rangle$. Let $\langle g, \gamma\rangle$ be the disjoint union of these models. Then the algebra induced by $\gamma$ on $g$ is easily seen to be isomorphic to $\mathcal{F}_{\Lambda}(k)$. There exists a modally saturated ultrapower $h$ such that $g \preccurlyeq h$ and $\left\langle h, \mathcal{F}_{\Lambda}(k)\right\rangle$ is a saturated generalized frame and so by the $\Delta$-elementarity of $\mathfrak{X} h \in \mathfrak{X}$. Now $\Lambda$ is $\Sigma \Delta$-elementary and so $h \models \Lambda$ and since $h \rightarrow \mathcal{F}_{\Lambda}(k)_{\sharp}$ we must have $\mathcal{F}_{\Lambda}(k)_{\sharp} \vDash \Lambda$. 


\section{The Methodology of Modal Logic}

With the notation and the general observations of the last three chapters we are now in a position to show via some examples how the generalizations establish a unifying view on modal logic. I would like to start with some remarks about the aims and tools of modal logic. We can distinguish two sorts of motivations for studying a subject: extrinsic and intrinsic motivations. The former have to do with 'applications' whilst the latter are generated by a desire to establish some inner organisation of the field itself. Completeness theory is a typical example of an extrinsically motivated field simply because of the connection between fmp alias $\mathfrak{F}$-completeness with decidability of a logic; and the latter property ranks high in the list of desiderata for a logic from a user's point of view. Correspondence theory is an example of an intrinsically motivated theory. The original aim was not to establish it for anything other than to answer questions that seemed good to ask in order to connect modal logic with established fields - in this case predicate logic and model theory. Note, for example, that if correspondence theory was founded to answer practical questions such as 'can we axiomatize the modal theory of such and such class of frames?' then surely one would have tried to come up with a characterization of the first-order correspondents of Sahlqvist formulas. Such a characterization has in fact never been given. The usefulness of correspondence theory can, however, be greatly improved if the following tacit assumptions are dropped.

- That $1^{s t}$-order notions rather than higher order ones are per se interesting, intuitive or useful.

- That $1^{s t}$-order correspondence is to be established on Kripke-frames.

We have to say that in [Benthem, 1983] these claims are never made but the questions (1) - (3) on p.13 do not make much sense otherwise. Note also that Chapter XIII of that book actually discusses correspondence and persistence in arbitrary classes of (generalized) frames but the connections are never worked out fully. We will challenge both assumption with some examples.

ExAmple 1. It is known that the axiom $\square p \rightarrow \square \square p$ corresponds to transitivity on Kripke-frames. Unfortunately this does not imply any completeness result for K4. (Observe that Kripke-elementary implies Kripke-persistence trivially, but completeness is still conditional Kripke-completeness so Prop. 6 does not help.) Thus it would be far better if 
such a result holds in generalized frames. It does not, for if we take $\mathcal{F}=\langle f,\{\emptyset,\{a, b, c\}\}\rangle$ and $\mathcal{G}=\langle g,\{\emptyset,\{x\}\}\rangle$ with $f, g$ as below, then $\mathcal{F}$ and $\mathcal{G}$ have the same modal theory since they support isomorphic set-algebras.

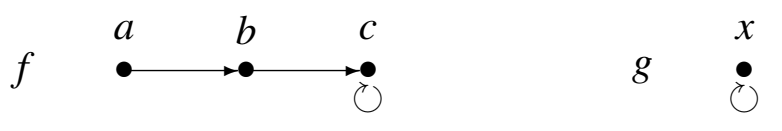

Since $g$ is transitive and we also have $\mathcal{G} \vDash \square p \rightarrow \square \square p$; this much is always true. Now we also have $\mathcal{F} \vDash \square p \rightarrow \square \square p$; but $\mathcal{F}$ is not transitive. Yet, if we assume that $\mathcal{G}$ is tight we can prove that $\mathcal{G} \vDash \square p \rightarrow \square \square p$ implies $\mathcal{G} \vDash(\forall x)\left(\forall y \triangleright^{2} x\right)(x \triangleleft y)$ - the other direction is true for all frames, as already said. Assume now $\mathcal{G} \not \models(\forall x)\left(\forall y \triangleright^{2} x\right)(x \triangleleft y)$; then there are points $r \triangleleft s \triangleleft t$ such that $r \Varangle t$. By tightness we have a valuation $\gamma$ such that $t \notin \gamma(p)$ but $r \in \gamma(\square p)$. Then $r \in \gamma(\diamond \diamond \neg p)$ and so $\langle\mathcal{G}, \gamma, r\rangle \vDash \square p \wedge \diamond \diamond \neg p$ from which $\mathcal{G} \nvdash \forall p \rightarrow \square \square p$. The class of tight frames is surely large enough since it includes all refined frames. Consequently, as every logic is $\mathfrak{T} \mathfrak{i}$-complete we can conclude that $\mathbf{K} \mathbf{4}$ is $\mathfrak{T} \mathfrak{i}$-complete, $\mathfrak{T} \mathfrak{i}$-elementary, whence $\mathfrak{T} \mathfrak{i}$-persistent and so Kripke-complete.

Of course we could have proved this result for descriptive frames; that would have been enough. But to have the result we proved is far better, because in practice one often has to construct certain generalized frames for a logic to see that certain formulae are consistent. One will surely appreciate then not having to create a descriptive frame but only a tight one!

ExAmple 2. Let $\mathcal{G}=\langle g, \mathbb{G}\rangle$ be a $\mathbf{K 4}$-frame. Call $\mathcal{G}$ separable if $\mathcal{G}$ is refined and for all $s \in g$ there is a $a_{s} \in \mathbb{G}$ such that for all $t \triangleright s$ either $t=s$ or $t \notin a_{s}$. Denote by $\mathfrak{S e p}$ the class of all transitive separable frames. In [Rautenberg, 1979] on p. 191 the question is asked whether every extension of S4 is Sep-complete. [Fine, 1974] had already answered this question positively. Indeed, he showed that every extension of $\mathbf{K} \mathbf{4}$ is $\mathfrak{S e p}$-complete. Why is this interesting? Because in the same paper he shows that every transitive logic of finite width if Sep-persistent and-violà-Kripke-complete.

ExAmple 3. Consider the classical cases of truly second order modal axioms, nameley the Gödel axiom and the Grzegorczyk axiom.

$$
\begin{array}{ll}
\text { G } & \square(\square p \rightarrow p) \rightarrow \square p \\
\text { Grz } & \square(\square(p \rightarrow \square p) \rightarrow p) \rightarrow p
\end{array}
$$


First of all, even though they do not correspond to a $1^{\text {st }}$-order property, they nevertheless do express rather simple properties namely to be transitive, (irreflexive and) without infinite ascending chains in the case of $\mathbf{G}$ and to be transitive reflexive and without strictly ascending chains in the case of Grz. Moreover, if we restrict our attention to finite frames the sun is shining again. For then $\mathbf{G}$ simply wants irreflexive and transitive frames and Grz reflexive, transitive frames without proper clusters (i. e. posets). Notice that these properties are even universal:

$$
\begin{array}{ll}
\text { G } & (\forall x)\left(\forall y \triangleright^{2} x\right)(x \triangleleft y),(\forall x)(x \not x) \\
\text { Grz } & (\forall x)\left(\forall y \triangleright^{2} x\right)(x \triangleleft y),(\forall x)(x \triangleleft x),(\forall x)(\forall y \triangleright x)(y=x \vee y \nless x)
\end{array}
$$

These logics are special cases of subframe logics which (i) are universal on finite Kripkeframes and (ii) have fmp, i.e. are $\mathfrak{F}$-complete ([Fine, 1985]). They are $1^{\text {st }}$-order on all frames exactly if they are determine a universal and positive condition on the finite frames iff they are natural i. e. $\mathfrak{R}$-persistent.

ExAmple 4. As so many axioms are not $1^{\text {st }}$-order there is a need to describe the geometrical content of an axiom in whatever terms but with notions that are as simple as possible. [Zakharyaschev, 1987] presents a real breakthrough in this direction. He presents a method to decompose any given axiom for a transitive logic into a finite set of so-called canonical formulas. The canonical formulas themselves are immediately connected with a geometric condition which is recoverable from their syntactic form and use notions such as (confinal) subreductions and closed domains. Clearly, ' $\mathcal{F}$ (confinally) subreduces to $\mathcal{G}^{\prime}$ is for finite $\mathcal{G}$ generally not $1^{\text {st }}$-order; but if it is it seems that then all canonical formulae $\alpha(G, \mathfrak{D}, \perp)$ are $1^{s t}$-order. To show the usefulness of this we turn to Example 2 again. Denote by $\mathfrak{S e p}^{b}$ the class of finitely generated separable frames. If $\mathcal{G} \in \mathfrak{S e p}^{b}$ is of finite width then $\mathcal{G}$ is without ascending chains and hence has the finite cover property (see [Fine, 1974]). If $\mathcal{F}$ is a finite frame and $\alpha(\mathcal{F}, \mathfrak{D}, \perp)$ is a canonical formula based on $\mathcal{F}$ then, as is not so hard to show, $\alpha(\mathcal{F}, \mathfrak{D}, \perp)$ is elementary in $\mathfrak{S e p}^{\mathfrak{b}_{\bullet}}$; for the proposition variables of $\alpha(\mathcal{F}, \mathfrak{D}, \perp)$ may without loss of generality be assumed to take only convex sets as values (minus some covering points); and they can be described using only the outer points of these sets. Now each set is covered by at most $n$ points in case of width $n$ and this proves modulo some handwaving that $\alpha(\mathcal{F}, \mathfrak{D}, \perp)$ has a $1^{\text {st }}$-order equivalent based on $2 n \times \sharp \mathcal{F}+1$ world-variables. If that is so, then all logics of width $n$ are $\mathfrak{S e p}^{\mathrm{b} \bullet}-\Delta$-elementary and hence $\mathfrak{S}_{\mathfrak{e}} \mathfrak{p}^{\mathrm{b}}$-persistent and so, once again, Kripke-complete. 


\section{General Correspondence}

Definition 9 Let $\mathfrak{X}$ be a class of frames and $\alpha, \beta \in \mathcal{L}^{e}$. We say that $\alpha$ corresponds with $\beta$ in $\mathfrak{X}$ and write $\alpha \leftrightarrow x \beta$ if $\mathfrak{X} \vDash(\forall \bar{p} \forall \bar{w})(\alpha \equiv \beta)$, where $\bar{p}, \bar{w}$ collect all free propositionand world-variables from $\alpha$ and $\beta$.

If we take $\mathfrak{X}$ to be $\mathfrak{K}$ and $\alpha=w \epsilon(\forall \bar{p}) Q$ then correspondence of $\alpha$ with $\beta \in \mathcal{L}^{f}$ in $\mathfrak{X}$ as defined here is exactly the classical correspondence problem. This section is devoted to the study of the interplay between invariance properties of formulae and their syntactic form. It will be pretty much a selection of results that can for example be found in [Benthem, 1984]; it cuts the classical correspondence problem from the far end telling us what properties are not definable, while later we will prove positive theorems giving characterizations of definable properties.

Let us consider the interplay between operators on classes of frames and preservation of validity. As explained in the first section, a map between e-models is uniquely defined by the map between the underlying generalized frames (which in turn is defined by the map between the underlying Kripke-frames). Various types of maps between generalized frames therefore define various operators on classes of generalized frames as well as triples defined over them. Such operators we call map-operators. For example, the surjective $\mathrm{p}$-morphisms define the operator $\mathrm{C}$ which, given a class $\mathfrak{X}$ of frames returns the class $\mathrm{C}(\mathfrak{X})$ of all p-morphic images (or contractions) of frames in $\mathfrak{X}$. Injective $\mathrm{p}$ morphisms define the operator $\mathrm{W}$, coproducts the operator CP. Moreover, we have the bidual B: $\mathcal{G} \mapsto\left(\mathcal{G}_{+}\right)^{+}$, the 'framification' $\mathrm{F}: \mathcal{G} \mapsto\left(\mathcal{G}_{\sharp}\right)^{\sharp}$ and the usual ultraproduct Up. Finally, a map $i: \mathcal{G} \rightarrow \mathcal{H}$ is a subalgebra if $i_{\sharp}$ is an isomorphism. The operator of subalgebras is denoted by $\mathrm{S}_{\mathrm{A}}$. All these map-operators are what [Sambin and Vaccaro, 1988] call weak contractions, which are defined as maps $p$ such that $p^{+}$is a homomorphism. The bidual map $w \mapsto \mathfrak{U}_{w}: \mathcal{G} \neg\left(\mathcal{G}_{+}\right)^{+}$is an example of a weak contraction that is not a p-morphism. Up presents an example of a non-map-operator. By convention, a mapoperator $\mathrm{O}$ collects all frames vz. e-models which are targets of a map for $\mathrm{O}$ with source in $\mathfrak{X}$; this defines $\mathrm{O} \mathfrak{X}$. This corresponds with the normal usage of these symbols except for the subframe operator where we have $\mathcal{G} \in \mathrm{W} \mathcal{H}$ iff $\mathcal{H}$ is a subframe of $\mathcal{G}$.

Definition 10 Let $\mathrm{O}$ be an operator on e-models and $\alpha \in \mathcal{L}^{e}$. We say that $\mathrm{O}$ preserves $\alpha$ in $\mathfrak{X}$ iffor all e-models $\mathcal{M}, \mathcal{N}$ from $\mathfrak{X}$ such that $\mathcal{N} \in \mathrm{O} \mathcal{M} \mathcal{M} \vDash \alpha \Rightarrow \mathcal{N} \vDash \alpha$. O reflects $\alpha$ in $\mathfrak{X}$ if $\mathrm{O}$ preserves $\sim \alpha$ in $\mathfrak{X}$ and $\mathrm{O}$ leaves $\alpha$ invariant if $\mathrm{O}$ both preserves and reflects $\alpha$. 
There are some simple facts one can collect about preservation.

Fact 11 If $\mathrm{O}$ preserves $\alpha$ and $\beta$ in $\mathfrak{X}$ then it also preserves $\alpha \& \beta$ and $\alpha \vee \beta$. Likewise for reflection and invariance. Moreover, $\mathrm{O}$ leaves $\alpha$ invariant iff it leaves $\sim \alpha$ invariant. Hence, operator invariance is closed under boolean combinations.

Fact 12 All map-operators defined above preserve $v \doteq w, v \triangleleft w$ and $v \in P$.

Proof. Suppose that $\mathrm{O}$ is defined from a type of map $p$. Let $p: \mathcal{G} \rightarrow \mathcal{H}$. Now let $\mathcal{M}=\langle\mathcal{G}, \gamma, \iota\rangle$ and $\mathcal{N}=\langle\mathcal{H}, \delta, \kappa\rangle$ be e-models and $p: \mathcal{M} \rightarrow \mathcal{N}$, that is, $\kappa=p \circ \iota, \gamma=p^{+} \circ \delta$. Then $\mathcal{M} \vDash v \doteq w \Rightarrow \mathcal{N} \vDash v \doteq w$ since $\iota(v)=\iota(w) \Rightarrow p \circ \iota(v)=p \circ \iota(w) \Rightarrow \kappa(v)=\kappa(w)$. Also $\mathcal{M} \vDash v \triangleleft w \Rightarrow \mathcal{N} \vDash v \triangleleft w$; for if $\iota(v) \triangleleft \iota(w)$ then $p \circ \iota(v) \triangleleft p \circ \iota(w)$ (since all maps considered are $\triangleleft$-homomorphic) and so $\kappa(v) \triangleleft \kappa(w)$. Finally, if $\mathcal{M} \vDash v \in P$ then $\iota(v) \in \bar{\gamma}(P)=\overline{p^{+} \circ \delta}(P)=p^{+} \circ \bar{\delta}(P)$ (for in all cases $p^{+}$is a homomorphism). Now $\kappa(v) \in \bar{\delta}(P)$ by definition of $p^{+}$and so $\mathcal{N} \vDash v \in P$.

We see that for the above result to hold, the underlying Kripke-map must be a $\triangleleft$-homomorphism and $p^{+}$an algebraic homomorphism. If $p$ is injective then all three formulae are in fact invariant under all map-operators. This can be sharpened considerably by the following

Theorem 13 If $\alpha$ is free of world-quantifiers then $\alpha$ is invariant under $\mathrm{W}, \mathrm{Cp}$ and $\mathrm{B}$. If $\alpha$ is free of proposition quantifiers then $\alpha$ is invariant under $\mathrm{F}$ and $\mathrm{SA}_{\mathrm{A}}$.

Proof. Note that all operators derive from maps which are injective. To prove the first claim note also that if $c: \mathcal{G} \neg \mathcal{H}$ is injective then $c^{+}$is onto. We can then simply assume that $\mathcal{G} \subseteq \mathcal{H}$. The proof is most easy if in tandem with our claim we also prove a 'locality principle' which states that if $\langle\mathcal{H}, \delta, \kappa\rangle \vDash \alpha$ then $\langle\mathcal{H}, a \cap \delta, \kappa\rangle \vDash \alpha$ for $a \cap \delta(p):=a \cap \delta(p)$ as long as range $(\delta) \subseteq a$. The proof is then by induction on $\alpha$. The ground clauses are covered by Fact 12 . The locality principle is also straightforward in these cases. Only the quantifier is not immediate. Thus let $\alpha=(\exists p) \beta$. Then

$$
\begin{aligned}
& \langle\mathcal{H}, \delta, \kappa\rangle \models(\exists p) \beta \\
\Leftrightarrow & \text { there is } \bar{\delta} \supseteq \delta \text { with } p \in \operatorname{dom}(\bar{\delta}) \text { and }\langle\mathcal{H}, \bar{\delta}, \kappa\rangle \vDash \beta \\
\Leftrightarrow & \text { there is } \bar{\delta} \supseteq \delta \text { with } p \in \operatorname{dom}(\bar{\delta}) \text { and }\langle\mathcal{H}, g \cap \bar{\delta}, \kappa\rangle \vDash \beta \\
\Leftrightarrow & \text { there is } \bar{\gamma} \supseteq \gamma \text { with } p \in \operatorname{dom}(\bar{\gamma}) \text { and }\langle\mathcal{G}, \bar{\gamma}, \iota\rangle \vDash \beta \\
\Leftrightarrow & \langle\mathcal{G}, \gamma, \iota\rangle \models \beta
\end{aligned}
$$


From this the locality for $\alpha$ is also immediate. For the second claim concerning the $\alpha$ 's which are free of proposition quantifiers observe that $\mathrm{F}$ and $\mathrm{S}_{\mathrm{A}}$ are based on maps which are Kripke-isomorphisms and therefore leave every $\mathcal{L}^{f}$ formula invariant. By Fact 12 the rest follows.

Fact 14 Let $\mathfrak{Y}=\mathrm{OX}$ and $\alpha \leftrightarrow \mathfrak{X} \beta$. Then if both $\alpha$ and $\beta$ are $\mathrm{O}$-invariant on $\mathfrak{Y}$, $\alpha \stackrel{\leftrightarrow}{ } \beta$.

Fact 15 If $\mathfrak{Y}=\mathrm{F} Y$ then $\alpha$ is invariant under $\mathrm{F}$ in $\mathfrak{Y}$ iff $\alpha$ is invariant under $\mathrm{S}_{\mathrm{A}}$ in $\mathfrak{Y}$.

The following theorems will be stated without proofs. They are more or less straightforward generalizations from [Benthem, 1984] and [Goldblatt, 1989]. First, call $\alpha$ quasielementary if $\alpha$ is free of propositional quantifiers. Call a class $\mathfrak{X}$ quasi-elementary if it is closed under framification and if $\mathfrak{X} \cap \mathfrak{K}$ is elementary.

Theorem 16 Let $\mathfrak{X}$ be quasi-elementary. Then $\alpha$ corresponds to a quasi-elementary $\beta$ in $\mathfrak{X}$ iff $\alpha$ is invariant under framification and preserved by Up in $\mathfrak{X} \cap \mathfrak{K}$. (

This theorem covers the remaining case of the Up operator. We can use it to prove a theorem of [Fine, 1975]. For note that $\mathfrak{R}$ is quasi-elementary, since it is determined by a universal second order sentence. Now in $\Re$ the sentence $\alpha=(\forall w)(\forall \bar{p})(w \in P)$ describes an elementary subclass of $\mathfrak{R}$ as can easily be checked using closure under Up as a criterion. If $\alpha$ is closed under framification, it is therefore equivalent to a first-order sentence.

Theorem 17 (Fine) Let $\boldsymbol{K}(P)$ be $\mathfrak{R}$-persistent. Then $\boldsymbol{K}(P)$ is $\mathfrak{R}$-elementary and a fortiori $\mathfrak{K}$-elementary.

With some more effort it is possible to prove that an $\Re$-persistent logic is $\Re$ - $\Delta$-elementary.

Theorem 18 Let $\mathfrak{X}$ be quasi-elementary and $\mathrm{W}^{-1}$-closed. Then $\alpha$ is reflected by $\mathrm{W}$ iff $\alpha$ correponds to an existentially restricted $\beta$. (

Theorem 19 Let $\mathfrak{X}$ be quasi-elementary and closed under $\mathrm{CP}$ and let $\alpha$ be quasi-elementary. Then $\alpha$ is preserved by $\mathrm{CP}$ iff $\alpha$ corresponds to a two-way restricted formula. ( 
Here, two-way restricted means that in addition to our normal restricted quantifiers we can also use the quantifiers $(\forall y \triangleleft x),(\exists y \triangleleft x)$ where $x$ is the restrictor.

Theorem 20 Let $\mathfrak{X}$ be quasi-elementary and closed under C. Let $\alpha$ be quasi-elementary. $\alpha$ is preserved by $\mathrm{C}$ iff $\alpha$ corresponds to a positive formula. (

Although the proof for these theorems does not allow to conclude that any combination of these syntactic classes and closure properties correspond in this way, most cumulative results turn out to be true nevertheless. A particularly valuable result is this.

Theorem 21 Let $\alpha$ be quasi-elementary and closed under $\mathrm{C}, \mathrm{CP}$ and $\mathrm{W}^{-1}$. Then $\alpha$ corresponds to a positive restricted formula in $\mathfrak{X}$ iff $\alpha$ is reflected by $\mathrm{W}$ and preserved by $\mathrm{C}, \mathrm{CP}$ in $\mathfrak{X}$. (

These results can also be reversed. This will then prove to be a major step towards correspondence theory. Let us say that a class $\mathfrak{X}$ is modally definable or modally axiomatic in $\mathfrak{Y}$ if there is a set $\Gamma \subseteq \mathcal{L}^{i}$ such that $\mathfrak{X}=M d_{\mathfrak{Y}}(\Gamma)$. Let us also call $\mathfrak{X}$ first-order definable or frame axiomatic in $\mathfrak{Y}$ if there is a set of sentences $\Gamma \subseteq \mathcal{L}^{f}$ such that $\mathfrak{X}=\operatorname{Md}_{\mathfrak{Y}}(\Gamma)$ and elementary in $\mathfrak{Y}$ if $\mathfrak{X}=M d_{\mathfrak{Y}}(\alpha)$ for some sentence $\alpha \in \mathcal{L}^{f}$.

Theorem 22 (Goldblatt) $\mathfrak{X}$ is modally axiomatic in $\mathfrak{G}$ iff $\mathfrak{X}$ is closed in $\mathfrak{G}$ under $\mathrm{C}, \mathrm{CP}$, $\mathrm{W}^{-1}, \mathrm{~B}$ and $\mathrm{B}^{-1}$. (

Theorem $23 \mathfrak{X}$ is elementary in $\mathfrak{G}$ iff $\mathfrak{X}$ is closed in $\mathfrak{G}$ under $\mathrm{F}, \mathrm{F}^{-1}$ and Up while $\mathfrak{G}-\mathfrak{X}$ is also closed under Up in $\mathfrak{G}$. (

Both can be boosted up to arbitrary classes $\mathfrak{Y}$ by requiring that $\mathfrak{X}$ is modally axiomatic (elementary) in $\mathfrak{Y}$ iff $\mathfrak{X}=\mathfrak{U} \cap \mathfrak{Y}$ for some modally axiomatic (elementary) $\mathfrak{U}$. However, instead of concentrating on classes of frames, it has proved best to consider classes of emodels instead. Here we take the closure properties of the frame classes as the definition for the classes of models. Thus a class $\mathfrak{X}$ of e-models is modally axiomatic in $\mathfrak{G}$ if it is closed under $\mathrm{C}, \mathrm{CP}, \mathrm{W}^{-1}, \mathrm{~B}$ and $\mathrm{B}^{-1}$ in $\mathfrak{G}$. Similarly, $\mathfrak{X}$ is elementary in $\mathfrak{G}$ if $\mathfrak{X}$ is closed under $\mathrm{F}, \mathrm{F}^{-1}$ and Up while its relative complement is also closed under Up; the definition is likewise upgraded for arbitrary classes. By Theorem $16, \mathfrak{X}$ is elementary in $\mathfrak{Y}$ iff $\mathfrak{X}=$ 
$M d_{\mathfrak{Y}}(\alpha)$ for some quasi-elementary $\alpha$. A similar characterization of modally axiomatic classes can be given as follows. Let us agree to call a formula of type $(\exists \bar{p}) \&\left\langle w_{i} \in Q_{i}\right.$ : $i \in n\rangle$ (or a formula equivalent to such a formula) a simple formula. Here it is not required that $\bar{p}$ binds all variables of the $Q_{i}$. Moreover, let us call a formula equivalent to a disjunction of simple formulae semi-simple. Semi-simple formulae are invariant under $\mathrm{W}, \mathrm{CP}, \mathrm{B}$ and $\mathrm{F}$ while only reflected by $\mathrm{C}$ and $\mathrm{S}$. Consequently one has the

Theorem 24 Let $\mathfrak{X}$ and $\mathfrak{Y}$ be classes of e-models. If $\mathfrak{X}=M d_{\mathfrak{Y}}(\Gamma)$ for some set $\Gamma$ of negations of semi-simple formulae then $\mathfrak{X}$ is modally axiomatic in $\mathfrak{Y}$.

We reckon that the converse is also true. Such facts have motivated the following definition.

Definition 25 Let $\alpha \in \mathcal{L}^{e}$ and $\mathfrak{X}$ be a class of frames. We say that $\alpha$ is internally describable in $\mathfrak{X}$ if $\alpha$ corresponds to some simple formula in the class of all e-models of $\mathfrak{X}$ and we say that $\alpha$ is internally semi-describable if $\alpha$ corresponds to a semi-simple formula. We say that $\alpha$ is internally definable (internally semi-definable) if $\sim \alpha$ is internally (semi-)describable.

\section{Three Ways of Proving Elementarity}

We are now approaching the question of internal definability in classes of frames. A main application of this will be (yet another) proof of Sahlqvist's Theorem. To see what is different and what is new let me walk you through a specific example in doing in via the method of substitutions, the topological way and finally method of internal descriptions.

The Method of Substitutions. (van Benthem) The axiom $\square p \rightarrow \square \square p$ imposes the second order condition $(\forall x)(\forall p)(x \in \square p \rightarrow \square \square p)$. Rewrite this as $(\forall x)(\forall p)(x \notin \square p \vee x \in$ $\square \square p)$ and then as $(\forall x)(\forall p)\left((\exists y \triangleright x)(y \in \neg p) \vee\left(\forall y \triangleright^{2} x\right)(y \in p)\right)$. Now take a specific set for $p$, namely the set $\{y: x \triangleleft y\}$. Then by slight abuse of the notation we can now deduce from the above condition $(\forall x)\left((\exists y \triangleright x)(y \notin\{y: x \triangleleft y\}) \vee\left(\forall y \triangleright^{2} x\right)(y \in\{y: x \triangleleft y\})\right)$ from which we get $(\forall x)\left((\exists y \triangleright x)(x \Varangle y) \vee\left(\forall y \triangleright^{2} x\right)(x \triangleleft y)\right)$, which reduces to $(\forall x)\left(\forall y \triangleright^{2} x\right)(x \triangleleft y)$. Thus if $\square p \rightarrow \square \square p$ is valid on a Kripke-frame then that frame is transitive. The other direction being always valid the two are therefore equivalent. Let me remark here that 
the success of this method lies in the possibility to have certain sets available in the frame (see the last chapter on decisive sets). Question: how do we know which substitution to choose? [Benthem, 1984] gives an answer but leaves us guessing how he found it.

The Topological Method. (Sambin, Vaccaro) We look at all instances of $\square p \rightarrow \square \square p$ in a frame; thus we consider $\bigcap\langle\mathbf{\square} a \rightarrow \mathbf{\square} a: a \in \mathbb{G}\rangle$. Modulo some rewriting we obtain that $w \in \mathbf{\square} a \rightarrow \mathbf{\square} a \Leftrightarrow r w \subseteq a \Rightarrow r r w \subseteq a$ where $r w=\{v: w \triangleleft v\}$. Thus $w \in \bigcap\langle\boldsymbol{\square} a \rightarrow \mathbf{\square} a: a \in \mathbb{G}\rangle \Leftrightarrow(\forall a \supseteq r w)(r r w \subseteq a) \Leftrightarrow \overline{r r w} \subseteq \overline{r w}$. Here, $\bar{a}$ denotes the topological closure of $a$ in $\langle g, \mathbb{G}\rangle$. Now, by a result of [Sambin and Vaccaro, 1988] Kripke-frames and descriptive frames are point closed and so $\overline{r w}=r w, \overline{r r w}=r r w$ and we obtain that $w \in \bigcap\langle\mathbf{\square} a \rightarrow \mathbf{\square} a: a \in \mathbb{G}\rangle \Leftrightarrow\left(\forall v \triangleright^{2} w\right)(w \triangleleft v)$ as desired. Note that this method as presented in [Sambin and Vaccaro, 1989] draws substantially from the topological results established in [Sambin and Vaccaro, 1988] which one has to master before being able to understand this proof. Secondly, it is not immediately clear how this generalizes to other classes of frames.

The Method of Internal Descriptions. The idea is once again to break the formula into parts whose meaning can separately be grasped. From there we assemble the $1^{s t}$ order correspondent. In order to do that we use two tricks. First, we do not prove that for every $\mathcal{G} \mathcal{G} \vDash P \Leftrightarrow \mathcal{G} \vDash \alpha$ but rather $\mathcal{G} \not \models P \Leftrightarrow \mathcal{G} \not \models \alpha$; which is the same, of course, but in practice much simpler to do because we can now reason by counterexample. Secondly, in order to decompose the formula completely and not be forced to stop halfway we consider $1^{\text {st }}$-order correspondents not for single formulas but for sequences of formulas (indeed, disjunctions over such sequences). This allows to have trans-world restrictions without any further ado. For example, if we want to state that two worlds are different then we can do this by naming a set $a$ that contains the one world but not the other. So, the pair $\langle p, \neg p\rangle$ corresponds in some sense to the set of pairs of different worlds. To perform the method with our example, let us show that

$$
\mathcal{G} \not \models \square p \rightarrow \square \square p \Leftrightarrow \mathcal{G} \not \models(\forall x)\left(\forall y \triangleright^{2} x\right)(x \triangleleft y) \text {. }
$$

How can this be done? Assume first that for some $\gamma$ and some $x, x \in \gamma(\square p \wedge \diamond \diamond \neg p)$. Then there is a $y \triangleright^{2} x$ such that $y \in \gamma(\neg p)$ and thus $x \not y$ since $x \in \gamma(\square p)$. The argument can be reversed on the condition of tightness. Note now that we need to look at two worlds $x, y$ rather than one world at a time. If we now use the convention $\alpha(\bar{w}) \leadsto\left\langle P_{i}: i \in n\right\rangle$ for $\bar{w}=w_{0}, \ldots, w_{n-1}$ to state that $\alpha$ holds of $\bar{w}$ exactly if for some $\gamma, w_{i} \in \gamma\left(P_{i}\right)$ for all $i \in n$; then we can perform the proof that $\square p \wedge \diamond \diamond \neg p \leftrightarrow\left(\exists y \triangleright^{2} w_{0}\right)\left(w_{0} \Varangle y\right)$ in tight 
frames as follows. Begin with $\langle\square p, \neg p\rangle \leadsto w_{0} \Varangle w_{1}$. Remember that $w_{0}$ is paired with $\square p$ and $w_{1}$ with $\neg p$ and thus this means that $w_{0} \vDash \square p$ and $w_{1} \vDash \neg p$ iff $w_{0} \Varangle w_{1}$. This is true exactly if $\mathcal{G}$ is tight. Now we 'calculate' as follows.

$$
\begin{aligned}
& \langle\square p, \neg p\rangle \quad \leadsto \quad w_{0} \Varangle w_{1} \\
& \langle\square p, \diamond \neg p\rangle \quad \leadsto \quad\left(\exists y \triangleright w_{1}\right)\left(w_{0} \Varangle y\right) \\
& \langle\square p, \diamond \diamond \neg p\rangle \leftrightarrow(\exists z \triangleright y)\left(\exists y \triangleright w_{1}\right)\left(w_{0} \not z\right) \\
& \langle\square p \wedge \diamond \diamond \neg p\rangle \leftrightarrow(\exists z \triangleright y)\left(\exists y \triangleright w_{0}\right)\left(w_{0} \Varangle z\right)
\end{aligned}
$$

Each step in the derivation is easily proved to be correct. The first two steps introduce $\diamond$ somewhere to the left and restricted $\exists$ to the right; in the last step two worlds get identified. There are two remarks on this method. The first is that $\alpha(\bar{w}) \leadsto\left\langle P_{i}: i \in n\right\rangle$ abbreviates the following $\mathcal{L}^{e}$-sentence

$$
(\forall \bar{w})\left(\alpha(\bar{w}) . \equiv .(\exists \bar{p})\left(\&\left\langle w_{i} \in P_{i}: i \in n\right\rangle\right)\right) .
$$

This sentence says nothing but that $\alpha$ is internally (semi-)describable! Thus the calculus we are presenting is nothing but a calculus to derive internal descriptions for elementary formulae. A second remark concerns the semantics of these sequences of propositions. We will talk about this topic in the following chapter and later return to the development of the calculus.

\section{Multi-frames and Multi-propositions}

Given an e-formule $\alpha$ with one free world-variable $w_{0}$, and given a frame $\mathcal{G}$ plus a valuation $\gamma$ with $p \operatorname{var}(\alpha) \subseteq \operatorname{dom}(\gamma) \alpha$ denotes a set in $\mathcal{G}$, namely the set of all $s \in \mathcal{G}$ such that $\langle\mathcal{G}, \gamma\rangle \vDash \alpha[s]$. This set is denoted by $\llbracket \alpha \rrbracket^{\langle\mathcal{G}, \gamma\rangle}$ or simply by $\llbracket \alpha \rrbracket$. $\llbracket \alpha \rrbracket$ is not necessarily internal. Similarly, an e-formula $\alpha$ with several free w-variables $\left\{w_{i}: i \in n\right\}$ denotes a set of $n$-tuples in $\langle\mathcal{G}, \gamma\rangle$. There is, however, a different way to look at the denotation of $\alpha$ namely by thinking of an $n$-tuple of worlds as a point in a new frame derived from $\mathcal{G}$. Such a frame, in which the worlds are the $n$-tuples of worlds from $\mathcal{G}$, we call a multi-frame based on $\mathcal{G}$. Let us start with the simplest example of a multi-frame, the bi-frame. From $\mathcal{G}$ we can form the frame $\mathcal{G} \otimes \mathcal{G}=\langle g \otimes g, \mathbb{G} \otimes \mathbb{G}\rangle$ where $g \otimes g=\langle g \times g, \triangleleft \times i d, i d \times \triangleleft\rangle$, a frame based on pairs of points from $g$ with two (!) accessibility relations; if the frame would 
be drawn in a plane, one might think of the first relation as the 'horizontal accessibility' and of the second as the 'vertical accessibility'. $\mathbb{G} \otimes \mathbb{G}$ is the carrier set of the set algebra corresponding to the tensor product of the algebras $\mathcal{G}_{+}$in the sense of boolean algebras. It consists of finite unions of 'rectangles' $a \times b$ with $a, b \in \mathbb{G}$. Since the underlying frame has two relations, $\mathbb{G} \otimes \mathbb{G}$ now has to be closed under two modal operators, namely

$$
\begin{aligned}
\diamond^{1}(a \times b) & =(\diamond a) \times b \\
\diamond^{2}(a \times b) & =a \times(\diamond b)
\end{aligned}
$$

It is clear that the boolean tensor product has these closure properties. We naturally have the projections which can be defined as follows; $[a \times b]_{1}=a$ and $[a \times b]_{2}=b$ and $[a \cup b]_{i}=[a]_{i} \cup[b]_{i}$. Similarly the $n$-frame ${ }^{n} \otimes \mathcal{G}:=\left\langle{ }^{n} \otimes g,{ }^{n} \otimes \mathbb{G}\right\rangle$ is defined. Just as ordinary frames can naturally interpret propositions, multi-frames are the natural habitat for multi-propositions. To continue the example, suppose that $\gamma$ is a valuation on $\mathcal{G}$; then $\gamma \otimes \gamma:\langle P, Q\rangle \mapsto \gamma(P) \otimes \gamma(Q)$ is a valuation on $\mathcal{G} \otimes \mathcal{G}$ which we call a bi-valuation. Let us agree to use $\otimes$ as a general sequence forming operator, so that we can write $P \otimes Q$ for $\langle P, Q\rangle$. We now change our language $\mathcal{L}^{i}$ to the language $\mathcal{L}^{\otimes}$ by adding $\otimes$ and replacing $\square, \diamond$ by $\square^{n}, \diamond^{n}, 0<n$, and call a formula of that language a multi-proposition. This definition is made sound for the intended interpretation by the convention to view a multiproposition $\mathfrak{M}$ as equivalent to $\mathfrak{M} \otimes \top$, so that for example $p . \wedge . q \otimes r$ is well-defined and equivalent to $p \otimes T . \wedge . q \otimes r$. Writing $\diamond$ for $\diamond^{1}$ and $\square$ for $\square^{1}$ we can note that any multiproposition $\mathfrak{M}$ can be converted into the form $\mathfrak{M} \equiv \bigvee\left\langle{ }^{n} \otimes P_{i j}: j \in n\right\rangle$ where $P_{i j}$ are modal propositions. (We write ${ }^{n} \otimes A_{i}$ for $\otimes\left\langle A_{i}: i \in n\right\rangle$, assuming that $n$ binds the running index i.) If we call a multi-proposition square if it is a sequence of propositions, then every multi-propositions is equivalent to a disjunction of squares. The length length $(\mathfrak{M})$ of a multi-proposition is the maximum of lengths of its disjuncts; and the length of a square is the number of propositions it contains, or, equivalently, $1+$ the number of $\otimes$ 's. Multipropositions correspond to semi-simple formulae in the same way as squares correspond to simple formulae. The nature of this correspondence will now be made explicit by deriving from each e-formula $\alpha$ in $n$ free variables a relational statement on $n$-sequences of worlds; this is done in much the same the way as Quine's formulation of predicate logic without variables. We will perform this translation in the most general form; let us assume that $\alpha$ has the free w-variables $\left\{w_{i}: i \in n\right\}$. $\alpha^{\dagger}$ is defined inductively as follows. 


$$
\begin{array}{ll}
\left(w_{i} \doteq w_{j}\right)^{\dagger} & =\Delta^{i j} \\
\left(w_{i} \triangleleft w_{j}\right)^{\dagger} & =\mathbb{B}^{i j} \\
\left(\left(\exists w_{i}\right) \alpha\right)^{\dagger} & =\exists^{i} \alpha^{\dagger} \\
\left(\left(\exists w_{i} \triangleright x\right) \alpha\left[x / w_{i}\right]\right)^{\dagger} & =\nabla^{i} \alpha^{\dagger} \\
\left(w_{i} \epsilon P\right)^{\dagger} & =\top \otimes \ldots \otimes P \otimes \ldots \otimes \top \\
(\alpha \& \beta)^{\dagger} & =\alpha^{\dagger} \cap \beta^{\dagger} \\
(\sim \alpha)^{\dagger} & =-\alpha^{\dagger} \\
((\exists p) \alpha)^{\dagger} & =\cup_{p} \alpha^{\dagger}
\end{array}
$$

To make this work, it is necessary to have also some operators manipulating the sequences of worlds. As in Quine's own proposal we use here the cycle $C\left({ }^{n} \otimes w_{i}\right)=w_{1} \otimes \ldots \otimes$ $w_{n-1} \otimes w_{0}$, the transposer $T^{i j}$, which permutes the positions $i$ and $j$ and finally $I^{i j}$ which identifies $i$ and $j$. But to make matters different we construe $I^{i j}$ as a map from ${ }^{n} \otimes \mathcal{G}$ to ${ }^{n-1} \otimes \mathcal{G}$. Finally, add the operator $D_{i}$, which iterates the $i$-th component; for example, $D_{0}\left(w_{0} \otimes w_{1}\right)=w_{0} \otimes w_{0} \otimes w_{1}$. It is not necessary to have an indexed set of equality and accessibility relations; indeed, they can be derived from a single one, namely $\Delta^{01}$ and $\AA^{01}$. It is for convenience also that we have included a version of a restricted quantifier which is non-contractive, that is, does not reduce the number of free variables. The contractive quantifier is readily defined fron the non-contractive one. The new symbols receive a straightforward interpretation in multi-frames. We put $\Delta^{i j}=\left\{\bar{s}: s_{i}=s_{j}\right\}$ and $\mathbb{\AA}{ }^{i j}=$ $\left\{\bar{s}: s_{i} \triangleleft s_{j}\right\}$. Moreover, $\diamond^{i} S=\left\{\bar{t}:(\exists \bar{s} \in S)\left(t_{i} \triangleleft s_{i}\right.\right.$ and $t_{j}=s_{j}$ for $\left.j \neq i\right\}$. The other symbols are straightforward; $\cap,-, \bigcup_{p}$ are intersection, complement and union over all possible valuations, and $\exists^{i} S$ is the set of all $n$-sequences $t$ such that $t$ derives from an $n+1$-sequence from $S$ by forgetting it's $i$-th component.

Given now an e-model $\mathcal{M}=\langle\mathcal{G}, \gamma, \iota\rangle$ with $\operatorname{dom}(\iota)=\left\{w_{i}: i \in n\right\}$ we can form an $n$-model ${ }^{n} \otimes \mathcal{M}=\left\langle{ }^{n} \otimes \mathcal{G},{ }^{n} \otimes \gamma,{ }^{n} \otimes \iota\right\rangle$ where ${ }^{n} \otimes \gamma$ acts as explained above and ${ }^{n} \otimes \iota$ is defined on a single variable $\bar{w}$ giving value $\left({ }^{n} \otimes \iota\right)(\bar{w})={ }^{n} \otimes \iota\left(w_{i}\right)$. The correspondence between $\alpha$ and $\alpha^{\dagger}$ is made explicit by the following proposition, which is easily proved by induction.

Proposition 26 Suppose that $\alpha$ has free variables $\left\{w_{i}: i \in n\right\}$. Then $\mathcal{M} \vDash \alpha$ iff ${ }^{n} \otimes \mathcal{M} \vDash$ $\bar{w} \epsilon \alpha^{\dagger}$. In short, $\llbracket \alpha \rrbracket=\alpha^{\dagger}$.

Note that the operators on variable sequences map internal sets into internal sets and can also naturally be interpreted as operators on multi-propositions. For if $\mathfrak{M}$ is a multi- 
proposition, then so are $C \mathfrak{M}, T^{i j} \mathfrak{M}, I^{i j} \mathfrak{M}, D^{i} \mathfrak{M}$. This is obvious for the permutations; for the other note that $I^{01}(P \otimes Q)=P \wedge Q$ and $I^{01}(\mathfrak{M} \vee \mathfrak{N})=I^{01} \mathfrak{M} \vee I^{01} \mathfrak{N}$ and also $D^{0}(P)=P \otimes P, D^{0}(\mathfrak{M} \vee \mathfrak{N})=D^{0} \mathfrak{M} \vee D^{0} \mathfrak{M}$. It is easily seen that if $\alpha$ is simple then $\alpha^{\dagger}=\bigcup_{\bar{p}} \mathfrak{S}$ for some square $\mathfrak{S}$ and that if $\alpha$ is semi-simple then $\alpha^{\dagger}=\bigcup_{\bar{p}} \mathfrak{M}$ for some multi-proposition $\mathfrak{M}$. Hence we can restate Def. 25 by saying that $\alpha$ is internally semidescribable (describable) in $\mathfrak{X}$ if for some multi-proposition (square) $\mathfrak{M} \mathfrak{X} \vDash \alpha^{\dagger}=\bigcup_{\bar{p}} \mathfrak{M}$.

\section{A Calculus for Internal Describability}

We will now develop a calculus for generating pairs of multi-propositions and quasielementary formulae corresponding in a given class of frames. For simplicity we assume that $\alpha$ contains no free proposition-variables, thus $\alpha \in \mathcal{L}^{f}$; it is then unnecessary to specify the set $\bar{p}$ and we can write $\bigcup \mathfrak{M}$ for $\bigcup_{\bar{p}} \mathfrak{M}$, where $\bar{p}$ is automatically the set of variables of $\mathfrak{M}$. Finally, we write $\alpha \leadsto \mathfrak{M}$ or $\mathfrak{M} \leadsto \alpha$ to say that $\mathfrak{M}$ internally describes $\alpha$.

Theorem 27 For every class $\mathfrak{X}$, internal describable formulae can be generated with the following rules:

$$
\begin{aligned}
& I_{\mathrm{T}} \text { true } \leadsto \mathrm{T} \\
& I_{\top} \text { true } \leadsto p \\
& I_{\vee} \frac{\alpha \leadsto \mathfrak{M} \quad \beta \rightsquigarrow \mathfrak{N}}{\alpha \vee \beta \rightsquigarrow \mathfrak{M} \vee \mathfrak{N}} \\
& C I_{\forall} \frac{\alpha \rightsquigarrow \mathfrak{C}^{i}}{\left(\forall x \triangleright w_{i}\right)\left(\alpha\left[x / w_{i}\right]\right) \leadsto \square^{i} \mathfrak{C}^{i}} \\
& I_{C} \frac{\alpha \rightsquigarrow \mathfrak{M}}{\alpha\left[w_{k+1(\bmod n)} / w_{k}\right] \rightsquigarrow C \mathfrak{M}} \\
& I_{I} \frac{\alpha \leadsto \mathfrak{M}}{\alpha\left[w_{j} / w_{i}, w_{j+k+1} / w_{j+k}\right] \leadsto I^{i j} \mathfrak{M}} \\
& S_{\sigma} \frac{\alpha \leadsto \mathfrak{M}^{\prime}}{\alpha \rightsquigarrow \mathfrak{M}^{\sigma}}
\end{aligned}
$$

$$
\begin{aligned}
& I_{\perp} \text { false } \leadsto \perp \\
& I_{\otimes} \frac{\alpha \rightsquigarrow \mathfrak{M} \quad \beta \rightsquigarrow \mathfrak{N}}{\alpha \wedge\left(\beta\left[w_{k+m} / w_{k}\right]\right) \rightsquigarrow \mathfrak{M} \dot{\otimes} \mathfrak{N}} \\
& I_{\exists} \frac{\alpha \rightsquigarrow \mathfrak{M}}{\left(\exists x \triangleright w_{i}\right)\left(\alpha\left[x / w_{i}\right]\right) \rightsquigarrow \diamond^{i} \mathfrak{M}} \\
& I_{\wedge} \frac{\alpha \leadsto \mathfrak{M} \quad \beta \rightsquigarrow \mathfrak{N}}{\alpha \wedge \beta \rightsquigarrow \mathfrak{M} \wedge \mathfrak{N}} \\
& I_{T} \frac{\alpha \leftrightarrow \mathfrak{M}}{\alpha\left[w_{i} / w_{j}, w_{j} / w_{i}\right] \rightsquigarrow T^{i j} \mathfrak{M}} \\
& I_{D} \frac{\alpha \leadsto \mathfrak{M}}{\alpha \wedge\left(\alpha\left[w_{n} / w_{n-1}\right]\right) \leftrightarrow D^{n-1} \mathfrak{M}} \\
& R_{\equiv} \frac{\alpha \rightsquigarrow \mathfrak{M} \quad \alpha \equiv \beta \quad \mathfrak{M} \equiv \mathfrak{N}}{\beta \leftrightarrow \mathfrak{N}}
\end{aligned}
$$

where $\alpha=\alpha\left(w_{0}, \ldots, w_{m-1}\right), \beta=\beta\left(w_{0} \ldots, w_{n-1}\right), \mathfrak{C}^{i}$ denotes a multi-proposition which is constant in the $i$-th component and $\dot{\otimes}(\dot{\wedge})$ is exactly like $\otimes(\wedge)$ but it replaces variables 
which occur both in $\mathfrak{M}$ and $\mathfrak{N}$ by different, new variables to avoid a collision in quantification; moreover, in $S_{\sigma}, \sigma$ is a substitution which can be inverted modulo equivalence, that is, $\sigma$ is an automorphism of the free $\mathbf{K}$-algebra. To save ink we have also assumed that $k$ is a running index, not a fixed number.

Proof. Straightforward checking. For example, if $\alpha \leadsto \mathfrak{M}$ and $\beta \leadsto \mathfrak{N}$ then $\alpha^{\dagger}=\bigcup \mathfrak{M}$ and $\beta^{\dagger}=\cup \mathfrak{N}$. Thus $\left.(\alpha \vee \beta)^{\dagger}=\alpha^{\dagger} \cup \beta^{\dagger}=\bigcup \mathfrak{M} \cup \cup \mathfrak{N}=\bigcup \mathfrak{M} \vee \mathfrak{N}\right)$. This shows the correctness of $I_{\mathrm{\vee}}$. Or, similarly, $\left(\alpha \wedge \beta\left[w_{k+m} / w_{k}\right]\right)^{\dagger}=\alpha^{\dagger} \otimes \beta^{\dagger}=\bigcup \mathfrak{M} \dot{\otimes} \cup \mathfrak{N}=\bigcup(\mathfrak{M} \dot{\otimes} \mathfrak{N})$, showing the correctness of $I_{\otimes}$.

Most of the rules explain themselves; note in particular that there is a special version of $S_{\sigma}$ which allows to swap negative and positive occurrences of a variable. Note also that we might replace $I_{\wedge}$ and $I_{\otimes}$ by rules in which it is presupposed that the antecedents are disjoint in the variables; with the substitution rules, the original rules can then be derived. The calculus with the above rules is denoted by Seq and sometimes referred to as the 'base calculus'. It specifies a notion of derivability of sequents $\alpha \leadsto \mathfrak{M}$. But we will also say that $\alpha$ is derivable if there is a multi-proposition $\mathfrak{M}$ such that $\alpha$ s $\mathfrak{M}$ is derivable and that $\mathfrak{M}$ is derivable if there is a $\alpha$ such that $\alpha \leadsto \mathfrak{M}$ is derivable. The idea of the subsequent theorems is to establish some connection between restricted classes of frames and additional rules or axioms valid in these classes. Below we give some easy examples of derivations in Seq.

EXAMPLE 5.

$$
\begin{array}{lrll}
I_{\top} & \text { true } & \rightsquigarrow & \top \\
I_{\exists} & \left(\exists x \triangleright x_{0}\right)(\text { true }) & \rightsquigarrow & \diamond \top
\end{array}
$$

Thus for a generalized frame $\mathcal{G} \vDash \square \perp \Leftrightarrow \mathcal{G} \vDash(\forall x) \sim(\exists y)(x \triangleleft y)$.

EXAMPLE 6.

$$
\begin{array}{lrll}
I_{\perp} & \text { false } & \longleftrightarrow & \perp \\
C I_{\forall} & \left(\forall x \triangleright x_{0}\right)(\text { false }) & \square & \square \perp
\end{array}
$$

Thus for every $\mathcal{G}, \mathcal{G} \vDash \diamond T \Leftrightarrow \mathcal{G} \vDash(\forall x)(\exists y)(x \triangleleft y)$. 


\section{EXAMPLE 7.}

$$
\begin{aligned}
& I_{\perp} \text { false } \leadsto \perp \\
& R_{\equiv} \text { false } \leadsto \square \perp \wedge \diamond \top \\
& R_{\equiv} \text { false } \leadsto \square p \wedge \square \neg p \wedge \diamond \top
\end{aligned}
$$

Thus every generalized frame satisfies $\mathcal{G} \vDash \diamond p \vee \diamond \neg p \vee \square \perp$; in other words the latter is a theorem of $\mathbf{K}$.

The applications of the base calculus seem to be rather limited; Theorem 31 shows that this is not an accident. We have to choose smaller classes to get better results. Note for the moment, however, the following fact.

Fact 28 If $\alpha\left(w_{0}\right)$ is built from formulae which are internally semi-describable in $\mathfrak{X}$ with the help of $\&, \vee$ and restricted $\exists$ then $\alpha$ is internally describable in $\mathfrak{X}$.

Proof. By the above rules, $\alpha$ is semi-describable. But since $\alpha\left(w_{0}\right)$ has only one free variable, $\alpha$ is also describable.

For restricted classes, somewhat better results can be obtained.

Proposition $29 w_{0} \neq w_{1}$ is internally describable in $\mathfrak{X}$ iff $\mathfrak{X} \subseteq \mathfrak{D} \mathfrak{f} . w_{0} \Varangle w_{1}$ is internally describable iff $\mathfrak{X} \subseteq \mathfrak{T} \mathfrak{i}$.

\section{Proof.}

$$
\begin{aligned}
& \mathcal{G} \in \mathfrak{D f} \Leftrightarrow \quad \mathcal{G} \vDash\left(\forall w_{0}\right)\left(\forall w_{1}\right)\left(w_{0} \doteq w_{1} \equiv(\forall p)\left(w_{0} \epsilon p \equiv w_{1} \epsilon p\right)\right) \\
& \Leftrightarrow \quad \mathcal{G} \vDash\left(\forall w_{0}\right)\left(\forall w_{1}\right)\left(w_{0} \neq w_{1} \equiv(\exists p)\left(w_{0} \epsilon p \wedge w_{1} \epsilon \neg p\right)\right) \\
& \Leftrightarrow{ }^{2} \otimes \mathcal{G} F\left(\forall w_{0} \otimes w_{1}\right)\left(w_{0} \otimes w_{1} \epsilon-\Delta \equiv(\exists p)\left(w_{0} \otimes w_{1} \epsilon p \otimes \neg p\right)\right) \\
& \Leftrightarrow{ }^{2} \otimes \mathcal{G} \vDash-\Delta \doteq \bigcup p \otimes \neg p \\
& \mathcal{G} \in \mathfrak{T} \mathfrak{i} \Leftrightarrow \quad \mathcal{G} \vDash\left(\forall w_{0}\right)\left(\forall w_{1}\right)\left(w_{0} \triangleleft w_{1} \equiv(\forall p)\left(w_{1} \epsilon p \supset w_{0} \epsilon \neg p\right)\right) \\
& \Leftrightarrow \quad \mathcal{G} \vDash\left(\forall w_{0}\right)\left(\forall w_{1}\right)\left(w_{0} \Varangle w_{1} \equiv(\exists p)\left(w_{1} \epsilon p \& w_{0} \epsilon \neg \nabla p\right)\right) \\
& \Leftrightarrow{ }^{2} \otimes \mathcal{G} \quad=\left(\forall w_{0} \otimes w_{1}\right)\left(w_{0} \otimes w_{1} \epsilon-\mathbb{B} \equiv(\exists p)\left(w_{0} \otimes w_{1} \epsilon \neg \nabla p \otimes p\right)\right) \\
& \Leftrightarrow{ }^{2} \otimes \mathcal{G} \quad=-\AA \doteq \bigcup(\square p) \otimes \neg p
\end{aligned}
$$


Theorem 30 If $\mathfrak{X} \subseteq \mathfrak{R}$ then the following axioms are valid in addition to the axioms and rules given above:

$$
I_{\neq} \quad w_{0} \not w_{1} \leftrightarrow p \otimes \neg p \quad I_{\phi} \quad w_{0} \not w_{1} \rightsquigarrow \square \square \otimes \neg p
$$

Thus every restricted, positive and universal $\alpha\left(w_{0}\right) \in \mathcal{L}^{f}$ is $\mathfrak{R}$-definable. $P$ is generated by $S$ eq $+I_{\neq}+I_{\Varangle}$ iff $P$ is equivalent to a formula built from constant propositions, boxed and unboxed variables and their negations using only $\wedge, \vee$ and $\diamond$.

\section{EXAMPLE 8.}

$$
\begin{array}{lrll}
I_{\neq} & p \otimes \neg p & \rightsquigarrow & x_{0} \neq x_{1} \\
I_{\exists} & \diamond p \otimes \neg p & \cdots & \left(\exists y \triangleright x_{0}\right)\left(y \neq x_{0}\right) \\
I_{\exists} & \diamond p \otimes \diamond \neg p & \rightsquigarrow & \left(\exists y \triangleright x_{0}\right)\left(\exists z \triangleright x_{1}\right)(y \neq z) \\
D & \diamond p \wedge \diamond \neg p & & \left(\exists y \triangleright x_{0}\right)\left(\exists z \triangleright x_{0}\right)(y \neq z)
\end{array}
$$

Thus a differentiated frame satisfies Alt $\mathbf{A l}_{\mathbf{1}}=\diamond p \rightarrow \square p$ iff $\triangleleft$ is quasi-functional i.e. $\mathcal{G} \vDash$ $(\forall x)(\forall y \triangleright x)(\forall z \triangleright x)(y \doteq z)$.

\section{EXAMPLE 9.}

$$
\begin{aligned}
& I_{\Varangle} \quad \square p \otimes \neg p \quad \leadsto \quad w_{0} \not w_{1} \\
& I_{T} \quad \neg p \otimes \square p \quad \leadsto \quad w_{1} \Varangle w_{0} \\
& I_{\sigma} \quad p \otimes \square \neg p \quad \leadsto \quad w_{1} \Varangle w_{0} \\
& I_{\exists} \quad p \otimes \diamond \square \neg p \quad \leadsto \quad\left(\exists y \triangleright w_{1}\right)\left(y \not w_{0}\right) \\
& D \quad p \wedge \diamond \square \neg p \quad \leadsto \quad\left(\exists y \triangleright w_{0}\right)\left(y \nless w_{0}\right)
\end{aligned}
$$

Thus a tight frame satisfies $\mathbf{B}=p \rightarrow \square \diamond p$ iff it is symmetric.

From the last theorem follows (modulo some handwaving) that all logics characterized by axioms of modal depth $\leq 1$ are $\Re$-elementary from which earlier results by van Benthem follow that these logics are $\mathfrak{K}$-elementary and $\mathfrak{K}$-complete (see [Benthem, 1984]). It seems that the calculi for $\mathfrak{G}, \mathfrak{D F}, \mathfrak{T} \mathfrak{i}$ and $\mathfrak{R}$ are complete; moreover, I conjecture that if $\mathfrak{X}$ is 
the class of frames in which a set $S$ of elementary formulae are internally definable, then $S e q+\left\{I_{\alpha}: \alpha \in S\right\}$ is complete for $\mathfrak{X}$. Note that by a result of [Chagrova, 1991] that ' $P$ corresponds to a $\forall$-sentence' is undecidable we cannot hope that the calculus $S e q+I_{\neq}+I_{\Varangle}$ is decidable if complete. The undecidability is clearly introduced by the rules $R_{\equiv}, S_{\sigma}$. With only $S_{\sigma}$ the derivable sequents are recursively enumerable since $\mathbf{K}$ is decidable; but with $R_{\equiv}$ this need no longer hold. With respect to the base calculus, completeness can be proved.

Theorem 31 Seq is complete for $\mathfrak{G}$. Thus a logic is $\mathfrak{G}$-persistent iff it is axiomatised by a set of constant axioms which express a constant first-order property of frames.

Proof. Suppose that $\Lambda$ is $\mathfrak{G}$-persistent and assume $\Lambda=\mathbf{K}(X)$ for some set $X$ of modal formulae. We already know that $\Lambda$ must be $\Delta$-elementary in $\mathfrak{R}$; thus let us say that $X$ corresponds to a set $\Gamma$ of first-order properties. By the fact that $\Gamma$ defines a modal class, we know that $\Gamma$ consists of positive and restricted formulae. Now let $\langle g, \mathbb{G}\rangle \vDash \Gamma$. Then $\langle g, \mathbb{H}\rangle \vDash \Gamma$, where $\mathbb{H}$ is the underlying set of the 0 -generated algebra in $\langle g, \mathbb{G}\rangle$. We can assume therefore that $\mathbb{G}$ is 0 -generated. Moreover, as $\alpha$ is restricted, we can assume that $g$ is generated by a single point called $s$. For the Kripke-frame $g$ we let $g^{+}$be the total unravelling of $g$. It consists of all paths starting at $s$. Formally, a path is a function $w_{n}: n=\{0,1, \ldots, n-1\} \rightarrow g$ such that $w_{n}(i) \triangleleft w_{n}(i+1)$ for all $i+1 \in n$. We call $w_{n}(0)$ the start point and $w_{n}(n-1)$ the end point of $w_{n}$. In $g^{+}$we have $v_{m} \triangleleft w_{n}$ for two paths iff $n=m+1, v_{m}(i)=w_{n}(i)$ for all $i \in m$ and $v_{m}(m-1) \triangleleft w_{n}(n-1)$. Thus $w_{n}$ extends $v_{m}$ by just one point which is accessible from its end point. The map $w_{n} \mapsto w_{n}(n-1)$ sending a path to its end point, is a p-morphism. $g^{+}$is a completely irreflexive, intransitive tree generated by $w_{1}: 0 \mapsto s$. It is clear that there exists a p-morphism $g^{\omega} \rightarrow g^{+}$from the frame $g^{\omega}$ which differs from $g^{+}$in that every point which has a successor in $g^{+}$branches infinitely often in $g^{\omega}$. Formally, $g^{\omega}$ can be obtained by the limit of the following construction. Starting at the generating point $w_{1}$ we replace all trees hanging off $w_{1}$ by infinitely many copies. This defines the frame $g_{1}^{+}$. If $g_{n}^{+}$is constructed, let $g_{n+1}^{+}$be the frame obtained by replacing each tree hanging off a point of depth $n$ by infinitely many copies. This completes the definition of $g_{n+1}^{+}$. Then put $g^{\omega}=g_{\omega}^{+}$. There is a p-morphism $g^{\omega} \rightarrow g^{+}$ and hence a p-morphism $g^{\omega} \rightarrow g$. Let $\mathcal{G}^{\omega}=\left\langle g^{\omega}, \mathbb{G}^{\omega}\right\rangle$ where $\mathbb{G}^{\omega}$ is the carrier of the 0 generated algebra on $g^{\omega}$. Since $\mathbb{G}^{\omega}$ is the preimage under this p-morphism of $2^{g}$ we must have $\mathcal{G} \vDash P$ and so $g^{\omega} \vDash \alpha$. Now we prove that this forces $\alpha$ to be constant for every $\alpha \in \Gamma$. Assuming that $\alpha$ is in prenex normal form and that every variable in $\alpha$ gets bound by exactly one quantifier we can say that $\alpha$ induces a tree ordering on the variables with 
root $r$. It is first of all easy to see that no subformula $v \doteq w$ can be ever be satisfied in $g^{\omega}$ if $v$ and $w$ are not of the same depth in that tree. Such a subformula we can replace by false. Consider the variables $x_{0}, \ldots, x_{n-1}$ of depth 1 in this tree and let $x_{i+1}$ be in the scope of the quantifier of $x_{i}$ for each $i \in n-1$. If there is an equation $v \doteq w$ with $v$ depending on $x_{i}$ and $w$ depending on $x_{j}, j>i$, then if $x_{j}$ is universally quantified, this equation can be replaced by false as there is always a choice for $x_{j}$ that can falsify it. But if $x_{j}$ is existentially quantified, there will be exactly one choice for $x_{j}$ that can make the equation true, namely if we chose the same value for $x_{j}$ as we did for $x_{i}$. Thus we replace a subformula $\left(\exists x_{j} \triangleright r\right) \phi$ by $\widetilde{\phi} \vee\left(\exists x_{j} \triangleright r\right) \bigvee\left\langle\phi\left[x_{i} / x_{j}\right]: i<j\right\rangle$ where $\widetilde{\phi}$ is obtained by replacing each equation $v \doteq w$ where $v$ depends on $x_{i}$ and $w$ on $x_{j}, i<j$, by false. Finally, all equations $x_{i} \doteq x_{i}$ are replaced by true. We do this for all $j$ from 1 to $n-1$ and get a formula which contains no $v \doteq w$ for $w, v$ dominated by different variables of depth 1. Now we can safely continue this procedure for all variables of depth 2 etc. yielding ultimately a formula free of equations.

The theorem slightly generalizes Lemma 13.2 of [Benthem, 1983]. The proof given here is much more complicated; however, it shows with a specific example that in general Theorem 21 follows from Theorems 18 and 19. For if $\alpha$ is restricted, so is $\sim \alpha$; and if $\sim \alpha$ is preserved under $\mathrm{C}^{-1}$ we can by the same unravelling argument show how to eliminate all positive equations, ending up with a negative, restricted formula.

\section{Sahlqvist's Theorem}

We want to show here that Sahlqvist's Theorem can be derived in a calculus that extends $S e q+I_{\neq}$by a special rule which is admissible for $\mathfrak{A}$, the class of approximation frames. This is more complicated than the earlier results. First some definitions. A multi-proposition $\mathfrak{M}$ is called strongly positive if for all frames $\mathcal{G}$ and all valuations $\gamma, \delta$, ${ }^{n} \otimes \mathcal{G} \vDash \mathfrak{M}[\gamma \cap \delta]=\mathfrak{M}[\gamma] \cap \mathfrak{M}[\delta] . \mathfrak{M}$ is called negative if for all frames $\mathcal{G}$ and all valuations $\gamma, \delta^{n} \otimes \mathcal{G} \vDash \mathfrak{M}[\gamma \cap \delta] \supseteq \mathfrak{M}[\gamma] \cup \mathfrak{M}[\delta]$. The following is easily established: if $\mathfrak{M}$ is composed from variables and constant propositions with the help of $\wedge, \square \square^{i}$ and $\otimes$ only, then $\mathfrak{M}$ is strongly positive. In this case $\mathfrak{M}$ is a square and each component can be written as a conjunction of formulae of type $\square^{\kappa} p_{i}$, with $i \in \omega$ and $\kappa \subset \omega, \kappa$ finite. If $\mathfrak{M}$ is built from constant propositions and negated proposition variables using only $\wedge, \vee, \diamond^{i}, \square^{i}$ and $\otimes$ then $\mathfrak{M}$ is negative. We call a multi-proposition $\mathfrak{M}$ a spone (from strongly positive negative) 
if it is a permutation of $\mathfrak{S} \otimes \mathfrak{N}$ where $\mathfrak{S}$ is strongly positive and $\mathfrak{N}$ is negative. If $\mathfrak{M}$ is a spone then all projections $[\mathfrak{M}]_{i}$ are either strongly positive or negative. Spones have the following rather crucial intersection property. Let $\mathcal{G}$ be a frame and $f_{s} \otimes f_{n} \subseteq{ }^{n} \otimes g$ a set with $f_{s}$ the part corresponding to $\mathfrak{S}$ and $f_{n}$ the part correspoding to $\mathfrak{N}$. Suppose that $f_{n}=g_{n} \cup h_{n}$ and that $f_{s} \otimes g_{n} \subseteq \mathfrak{S} \otimes \mathfrak{N}[\gamma]$ as well as $f_{s} \otimes h_{n} \subseteq \mathfrak{S} \otimes \mathfrak{N}[\delta]$. Then $f_{s} \otimes f_{n} \subseteq \mathfrak{S} \otimes \mathfrak{N}[\gamma \cap \delta]$. An infinitary analogue holds, if the frame allows infinite intersections.

Proposition 32 In $\mathfrak{A}$ the following rule is admissible for square spones.

$$
S I_{\forall} \frac{\alpha \rightsquigarrow \mathfrak{S} \otimes \mathfrak{N}}{\left(\forall x \triangleright w_{k}\right)\left(\alpha\left[x / w_{k}\right]\right) \rightsquigarrow \square^{k} \mathfrak{S} \otimes \mathfrak{N}} \quad k \geq \text { length(S) }
$$

Proof. For all $\gamma: \mathfrak{S} \otimes \mathfrak{N}[\gamma] \subseteq \alpha^{\dagger}$ by assumption and so $\square^{k} \mathfrak{S} \otimes \mathfrak{N}[\gamma] \subseteq \square^{k} \alpha^{\dagger}$ and consequently $\cup \square^{k} \mathfrak{S} \otimes \mathfrak{N} \subseteq \square^{k} \alpha^{\dagger}$. The converse inclusion is a bit trickier. For simplicity of notation let $k=$ length(S) + length $(\mathfrak{N})-1$. Take any ${ }^{n} \otimes s \in \alpha^{\dagger}$. Form the set $s_{k}^{\uparrow}=$ $\left\{t: s_{k} \triangleleft t\right\}$ and $S=\left\{s_{0}\right\} \otimes\left\{s_{1}\right\} \otimes \ldots \otimes s_{k}^{\uparrow}$. For each $x \in S$ there is a valuation $\gamma_{x}$ such that $x \in \mathfrak{S} \otimes \mathfrak{N}\left[\gamma_{x}\right]$. If we have a Kripke-frame we put $\gamma=\bigcap\left\langle\gamma_{x}: x \in S\right\rangle$ and by the intersection property ${ }^{n} \otimes s \in \square^{k} \mathfrak{S} \otimes \mathfrak{N}[\gamma]$. But if our frame is continuous, take the following construction. For a finite set $f \subseteq S$ we put $\gamma_{f}=\bigcap\left\langle\gamma_{x}: x \in f\right\rangle$. Then $f \subseteq \mathfrak{S} \otimes \mathfrak{N}\left[\gamma_{f}\right]$. Let $\mathcal{F}$ be the set of finite subsets of $S$; then $\Delta=\left(\gamma_{f}\right)_{f \in \mathcal{F}}$ is a downward going family of valuations. Hence $\mathfrak{S}[\Delta]$ and $\mathfrak{N}$ are downward vz. upward going families of sets and by continuity of the frame we have ${ }^{n} \otimes s \in \square^{k} S \subseteq \square^{k} \mathfrak{S} \otimes \mathfrak{N}[\lim \Delta]=\lim \square^{k} \mathfrak{S} \otimes \mathfrak{N}[\Delta] \subseteq$ $\cup \square^{k} \mathfrak{S} \otimes \mathfrak{N}$.

Let us now concentrate on the class of differentiated approximation frames, which is $\mathfrak{K} \cup \mathfrak{E} \cap \mathfrak{D F}$. In this class all of the abovementioned rules are valid and we denote by $\mathrm{Seq}^{+}$ the calculus $S e q+S I_{\forall}+I_{\neq}$. Our aim is to show that all square spones are derivable. We start with a slight reformulation of the original calculus $S e q+S I_{\forall}$. Given a spone $\mathfrak{M}$ we can assign a signature $\sigma_{\mathfrak{M}}$ to $\mathfrak{M}$ which is a sequence of $s$ and $n$ depending on whether $\mathfrak{M}_{i}$ is strongly positive or negative. It is especially helpful to write down a calculus for spones with identical signature. As it turns out, $S I_{\forall}$ and $I_{\exists}$ do not change the signature, neither does $I_{\wedge}$. However, $I_{\vee}$ is problematic since the consequent is not a square spone. In order to remedy this, we introduce the following specialized rules for square spones. 


$$
\begin{aligned}
& S I_{\wedge} \frac{\mathfrak{S} \otimes \mathfrak{M} \leadsto \alpha(\bar{w}, \bar{x}) \quad \mathfrak{T} \otimes \mathfrak{N} \leadsto \beta(\bar{w}, \bar{x})}{\mathfrak{S} \wedge \mathfrak{T}^{\sigma} \otimes \mathfrak{M} \wedge \mathfrak{N}^{\sigma} \leadsto(\alpha \& \beta)(\bar{w}, \bar{x})} \\
& S I_{\vee} \frac{\mathfrak{S} \otimes \mathfrak{M} \leadsto \alpha(\bar{w}, \bar{x}) \quad \mathfrak{T} \otimes \mathfrak{N} \leadsto \beta(\bar{w}, \bar{x})}{\mathfrak{S} \wedge \mathfrak{T}^{\sigma} \otimes \mathfrak{M} \vee \mathfrak{N}^{\sigma} \leadsto(\alpha \vee \beta)(\bar{w}, \bar{x})} \\
& S I_{\otimes} \frac{\mathfrak{S} \otimes \mathfrak{M} \leadsto \alpha(\bar{w}, \bar{x}) \quad \mathfrak{T} \otimes N \leadsto \beta\left(\bar{w}, x_{0}\right)}{\mathfrak{S} \wedge \mathfrak{T}^{\sigma} \otimes \mathfrak{M} \otimes N^{\sigma} \leadsto \alpha(\bar{w}, \bar{x}) \& \beta\left(\bar{w}, x_{n}\right)}
\end{aligned}
$$

Here all multi-formulas are spones of same signature, except for $S I_{\otimes}$; also all resulting spones are square with the exception of $S I_{\vee}$ when the signature contains more than one $n$. This will cause some complications. Moreover, we have used the convention to name the variables $w_{i}$ if they correspond to the $i$-th position in $\mathfrak{S}$ and $x_{j}$ if they correspond to the $j$-th position in the negative spone. In addition, $\sigma$ is assumed to be any renaming of variables to make $\mathfrak{S}$ and $\mathfrak{T}$ variable disjoint in case they are not equal. So, if $\mathfrak{S}=\mathfrak{T}$ then the consequent is $\mathfrak{S} \wedge \mathfrak{S}$. In that case we stipulate that the strongly positive part of the consequent of $S I_{\wedge}$ and $S I_{\vee}$ is $\mathfrak{S}$. (An application of $R_{\equiv}$ can derive this.) For the quantifiers we take $S I_{\exists}$ to be just $I_{\exists}$ and $S I_{\forall}$ as above so they are admissible in $S e q+S I_{\forall}$. To see that the three other rules are admissible, observe that from $p \leadsto$ true is derivable $\mathfrak{S} \otimes T \leadsto \mu$ for some constant $\mu$ containing only \& and $\forall$ and true; thus $\mu \equiv$ true and so with $R_{\equiv}$ we can derive $\mathfrak{S} \otimes \top \leadsto$ true and likewise $\mathfrak{T}^{\sigma} \otimes \top \leadsto$ true. Now we conclude

$$
\frac{\mathfrak{S} \otimes \mathfrak{M} \leadsto \alpha \quad \mathfrak{T}^{\sigma} \otimes \mathbf{T} \leadsto \text { true }}{\mathfrak{S} \wedge \mathfrak{T}^{\sigma} \otimes \mathfrak{M} \leadsto \alpha} \frac{\frac{\mathfrak{T} \otimes \mathfrak{N} \leadsto \beta}{\mathfrak{T}^{\sigma} \otimes \mathfrak{N}^{\sigma} \leadsto \beta} \mathfrak{S} \otimes \mathbf{T} \leadsto \text { true }}{\mathfrak{S} \wedge \mathfrak{T}^{\sigma} \otimes \mathfrak{N}^{\sigma} \leadsto \beta}
$$

from which by $I_{\wedge}$ vZ. $I_{\vee}$ the desired conclusion follows. $S I_{\otimes}$ can replaced by $I_{\otimes}$ followed by a sequence of $I_{I}$. Note also that every derivation with $S I_{\otimes}$ can be replaced by a derivation with $S I_{\otimes}$ being the last rule applied (i. e. applications if $I_{\otimes}$ can be postponed). Since $S I_{\wedge}, S I_{\vee}, S I_{\otimes}, S I_{\exists}$ and $S I_{\forall}$ are derivable in $S e q^{+}$we know that if $S I_{\Varangle}$ is also derivable, then all spones are derivable.

$$
S I_{\Varangle} \quad{ }^{n} \otimes \square \square^{\kappa_{i}} p . \otimes . \neg p \leadsto \&\left\langle w_{i} \not \not^{\kappa_{i}} x_{0}: i \in n\right\rangle
$$

For by $S I_{\otimes}$, proving $\mathfrak{S} \otimes{ }^{n} \otimes N_{i}$ reduces to proving $\mathfrak{S} \otimes N_{i}$ for each $i$ and by the other rules this further reduces to proving $\mathfrak{S} \otimes \neg p$. By $R_{\equiv}$ and the fact that $\mathfrak{S}$ is equivalent to a 
conjunction of spones of type ${ }^{n} \otimes \square{ }^{\kappa_{i}} p$, using the derivability of ${ }^{n} \otimes \square \square^{\kappa_{i}} q \otimes \neg p \leftrightarrow$ true for $p \neq q$ we see indeed that everything can be derived from $S I_{\not \downarrow}$. But now ${ }^{n} \otimes \square{ }^{\kappa_{i}} p . \otimes . \neg p$ can be derived by $S_{\sigma}$ and $R_{\equiv}$ from ${ }^{n} \otimes \square^{\kappa_{i}} \neg p . \otimes . p$ using the substitution $p \mapsto \neg p$. But ${ }^{n} \otimes \square{ }^{K_{i}} \neg p . \otimes . p$ is a square spone and can be derived by the $S I$-rules from $p \otimes \neg p$, which is an axiom of $S e q^{+}$. Thus in $S e q^{+}$all square spones can be derived.

Theorem 33 (Sahlqvist) If $P$ is created from strongly positive and negative propositions using only $\wedge, \vee$ and $\diamond$ then $\boldsymbol{K}(\neg P)$ is (locally) $\mathfrak{A} \cap \mathfrak{D f}$-persistent and $\mathfrak{A} \cap \mathfrak{D} \mathfrak{f}$-elementary. In particular, $\boldsymbol{K}(\neg P)$ is locally $\mathfrak{D}$-persistent and $\mathfrak{K}$-elementary.

Example 10. We derive a less well-known axiom in $S e q^{+}$. This logic is called $\mathbf{M k}^{3^{\prime}}$ in [Hughes and Cresswell, 1984]. This logic is Sahlqvist as is easily seen, but it incidentally fails to have fmp.

$\mathbf{M k}^{3^{\prime}} \quad q \wedge \square p \rightarrow \diamond(\square \square p . \wedge . \diamond q)$

$$
\begin{aligned}
& 1: S I_{\Varangle} \quad q \otimes \square p \otimes \neg p \quad \leadsto \quad w_{1} \Varangle x_{0} \\
& 2: 2 \times S I_{\exists} \quad q \otimes \square p \otimes \diamond \diamond \neg p \quad \leadsto \quad\left(\exists y \triangleright^{2} x_{0}\right)\left(w_{1} \not y\right) \\
& \text { 3:SI } \quad q \otimes \square p \otimes \square \neg q \quad \leadsto \quad x_{0} \nless w_{0} \\
& 4: S I_{\vee}(2,3) \quad q \otimes \square p \otimes \diamond \diamond p \vee \square \neg q \quad \leadsto \quad\left(\exists y \triangleright^{2} x_{0}\right)\left(w_{1} \Varangle y\right) \vee x_{0} \Varangle w_{0} \\
& 5: S I_{\vee} \quad q \otimes \square p \otimes \square(\diamond \diamond \neg p \vee \square \neg q) \quad \leadsto\left(\forall z \triangleright x_{0}\right)\left[\left(\exists y \triangleright^{2} z\right)\left(w_{1} \Varangle y\right) \vee z \nless w_{0}\right] \\
& 6: 2 \times D \quad q \wedge \square p \wedge \square(\diamond \diamond \neg p \vee \square \neg q) \quad \longleftrightarrow \quad\left(\forall z \triangleright w_{0}\right)\left[\left(\exists y \triangleright^{2} z\right)\left(w_{0} \Varangle y\right) \vee z \nless w_{0}\right]
\end{aligned}
$$

Hence if $\mathcal{G}$ is descriptive or Kripke, it satisfies $\mathbf{M k}^{\mathbf{3}^{\prime}}$ iff it satisfies $(\forall w)(\exists z \triangleright w)\left[\left(\forall y \triangleright^{2}\right.\right.$ $z)(w \triangleleft y) \wedge z \triangleleft w]$.

The statement of Thm. 33 is not quite optimal in the sense that we can exchange positive and negative occurrences of a variable. The seemingly best possible characterization is this: $P$ satisfies the theorem also if for every variable $p$ either (i) every subformula of type $\square Q$ which is positive in $p$ is also strongly positive in $p$ or (ii) every subformula of type $\square Q$ which is negative in $p$ is strongly negative in $p$. One may conclude that in face of the fact that S4.1 is $\mathfrak{D}$-persistent and elementary, Sahlqvist's Theorem is not exhaustive. But such a conclusion is not obvious in the present formulation of the theorem since we speak about local persistence. The full story is indeed rather complicated. Let us write $\mathfrak{X}^{b}$ for the class of finitely generated $\mathfrak{X}$-frames. Then, using the fact that transitive $\mathfrak{R}^{b}$-frames are top-heavy ([Fine, 1985]) and that there are a finite number of points of given finite depth we conclude that $\mathbf{S 4 . 1}$ although not locally $\mathfrak{D}$-persistent is locally $\mathfrak{R}^{b}$-persistent 
and hence also locally $\mathfrak{D}^{b}$-persistent (see [Kracht, 1991]). The latter seems to hold for all extensions of $\mathbf{K 4}$ which are characterized by modal reduction principles; and since the finitely generated descriptive frames are a total class, completeness follows. But I risk the bet that none of reduction principles which escape Sahlqvist's Theorem are locally $\mathfrak{D}$-persistent-not even in transitive frames.

We are now going to characterize all those elementary formulae which are $\mathrm{Seq}^{+}-$ derivable. This is done in three steps.

STEP 1. $\alpha\left(\bar{w}, x_{0}\right) \leadsto \mathfrak{S} \otimes N$ iff $\alpha\left(\bar{w}, x_{0}\right)$ is equivalent to a formula $\beta\left(\bar{w}, x_{0}\right)$ in which every subformula $\gamma$ is of type $\gamma(\bar{w}, z)$ for some $z$. This is easily shown by induction on the $S I$ calculus. Note that in this case we use spones with signature $\bar{s} \otimes n$ and therefore all rules may be applied without restrictions and yield square spones. The $\alpha$ thus obtained are said to be of type 1 .

STEP 2. $\alpha(\bar{w}, \bar{x}) \leadsto \mathfrak{S} \otimes \mathfrak{N}$ for some spone $\mathfrak{S} \otimes \mathfrak{N}$ iff $\alpha(\bar{w}, \bar{x})$ is composed from $w_{i} \not^{\kappa} z, z \not^{\kappa}$ $w_{j}, w_{i} \Varangle^{\kappa} w_{j}$ with the help of $\&, \vee$ and restricted $\forall, \exists$ with restrictors not from $\bar{w}$. To see this we show that such $\alpha$, which we call type 2 formulae, are (equivalent to) a disjunction of conjunctions of type 1 formulae and thus clearly derivable using $S I_{\wedge}, S I_{\otimes}$ and $S I_{\vee}$ on type 1 formulae; on the other hand, a derivable formula is surely of this form-as can inductively be shown.

The argument is as follows. Take a subformula $\phi=(\forall z \triangleright y) \psi$ of minimal rank such that $\phi$ is not of type 1 . Rewrite $\psi$ as a conjunction of disjunctions of quantified formulae and distribute the quantifier over the conjuncts. Then $\phi \equiv \&\left\langle(\forall z \triangleright y) \psi_{i}: i \in m\right\rangle$ where each $\psi_{i}$ is a disjunction of type 1 formulae $\chi_{i j}(\bar{w}, x)$. If $x \neq z$ then we may move $\chi_{i j}$ out of the scope the quantifier; thus we have arranged it that $\phi$ is a conjunction of disjunction of type 1 formulae. A dual argument is used for the existential quantifier. By induction on the quantifier rank we show that $\alpha$ is equivalent to a formula in which every subformula $(\forall z \triangleright y) \beta,(\exists z \triangleright y) \beta$ is of type 1 , as required.

STEP 3. We know that any $\alpha(\bar{w}, \bar{x})$ which is composed from type 2 formulae with the help of $\&, \vee$ and restricted $\exists$ is derivable in $\mathrm{Seq}^{+}$. Call such formulas type 3 formulas. The only difference with a type 2 formula is that the $w$-variables may now be bound variables-but only by existentials. Now call a variable $v$

inherently existential in $\alpha$ if either (i) $v$ is free in $\alpha$ or (ii) $\alpha \equiv(\exists x \triangleright y) \beta$ and $v$ is inherently existential in $\beta$. In other words, if $\alpha$ is in prenex normal form, $v$ is inherently 
existential iff it is not in the scope of a universal quantifier. Similarly inherently universal is defined. Then $\alpha$ is of type 3 iff it is composed from formulae of type $y \not z$ such that either $y$ or $z$ is inherently existential.

Theorem $34 \alpha$ is the elementary equivalent of a Sahlqvist formula iff $\alpha$ is equivalent to a positive, restricted formula in which every subformula $y \triangleleft^{\kappa} z$ contains at least one inherently universal variable.

Of course, all universal fornulae satisfy this condition. But also $(\forall v)(\forall w \triangleright v)(\forall x \triangleright v)(\exists y \triangleright$ $w)(x \triangleleft y)$ while $(\forall v)(\exists w \triangleright v)(\forall x \triangleright w)(x \doteq w)$ does not; of course, to see that a formula is not of the required syntactic form is not enough to show that the formula is not at all equivalent to a Sahlqvist formula. But with the formula just given this is indeed so.

We demonstrate the proof of the last theorem by a concrete example; we look at a formula which is a slight variation on the $\mathbf{M k}^{3^{\prime}}$-formula, namely

$$
\left.(\forall w)(\exists z \triangleright w)\left[\left(\forall y \triangleright^{2} w\right)(w \triangleleft y) \& z \triangleleft w\right)\right]
$$

We want to show that the negation of this formula is derivable.

$$
\left.\left(\exists w_{0}\right)\left(\forall z \triangleright w_{0}\right)\left[\left(\exists y \triangleright^{2} w_{0}\right)\left(w_{0} \Varangle y\right) \vee z \not w_{0}\right)\right]
$$

Note that only $w_{0}$ is inherently existential and since all subformulas contain $w_{0}$, the conditions are met and we should be able to derive this formula. We now reduce it from type 3 to type 2 by disintegrating $w_{0}$ into $x_{0}$ and $w_{0}$; for by the rule $I_{D}$ it is enough to derive a formula where some $w_{0}$ are replaced by $x_{0}$. By this we can get a formula in which $w_{0}$ is no longer a restrictor.

$$
\left.\left(\forall z \triangleright x_{0}\right)\left[\left(\exists y \triangleright^{2} x_{0}\right)(w) \nless y\right) \vee z \Varangle w_{0}\right]
$$

Note that the substitution $w_{0} / x_{0}$ gives us back the original formula. This formula is now of type 2 and should correspond with a spone. In fact, notice that the universal quantifier can be pushed inside.

$$
\left(\forall y \triangleright^{2} x_{0}\right)\left(w_{0} \nless y\right) \vee\left(\forall z \triangleright x_{0}\right)\left(z \nless w_{0}\right)
$$

This is now a disjunction of type 1 formulae, namely $\left(\exists y \nless x_{0}\right)\left(w_{0} \Varangle y\right)$ and $\left(\forall z \triangleright x_{0}\right)(z \Varangle$ $\left.w_{0}\right)$. The former is derived from $w_{0} \Varangle x_{0} \leadsto \square p \otimes \neg p$ by two steps of $S I_{\exists}$ getting us 
$\left(\exists y \Varangle x_{0}\right)\left(w_{0} \Varangle y\right) \leftrightarrow \square p \otimes \diamond \diamond \neg p$

The latter is derived from $p \otimes \neg p \longleftrightarrow w_{0} \neq x_{0}$ by two $S I_{\forall}$ yielding

$\left(\forall z \triangleright x_{0}\right)\left(z \nless w_{0}\right) \rightsquigarrow p \otimes \square \square \neg p$

Applying $S I_{\vee}$ we get

$\left(\exists y \triangleright^{2} x_{0}\right)\left(w_{0} \not y\right) \vee\left(\forall z \triangleright x_{0}\right)\left(z \not w_{0}\right) \leadsto q \wedge \square p \otimes \diamond \diamond \neg p \vee \square \square \neg q$

Finally, by $I_{D}$ and $R_{\equiv}$

$$
\left(\forall z \triangleright w_{0}\right)\left[\left(\exists y \triangleright^{2} w_{0}\right)\left(w_{0} \nless y\right) \vee z \nless w_{0}\right] \rightsquigarrow q \wedge \square p \wedge(\diamond \diamond \neg p \vee \square \square \neg q)
$$

\section{Topology and Decisive Sets}

There is yet another way to think about these results, which derives from the work of Sambin and Vaccaro. The thrust of it is to reduce the problem of internal describability in a class $\mathfrak{X}$ to internal describability in Kripke-frames. We do this by thinking of an arbitrary frame $\mathcal{G}$ simply as if it were the Kripke-frame $\left(\mathcal{G}_{\sharp}\right)^{\sharp}$ and asking ourselves first which sets we need or want to have assigned to $\bar{p}$ to make the equation $\alpha^{\dagger}=\bigcup_{\bar{p}} \mathfrak{M}$ true. We call these sets decisive for $\alpha$ (or $\mathfrak{M}$ ). The decisive sets are the equivalent of the substitutions in the 'method of substitutions' designed in [Benthem, 1984] and explained briefly above. Only after that we worry about the question whether these sets are internal. For example, $-\Delta=\bigcup p \otimes \neg p$ can be made true if only all atomic valuations i.e. valuations of type $p \mapsto\{w\}$ are chosen. The interesting fact is that although these singleton sets are mostly not internal, they can nevertheless be approximated by a family of sets. If we were to use topology, let the frame $\mathcal{G}$ serve as the base of clopen (= closed and open) sets of a topological space denoted by $\mathcal{G}^{\circ}$; then a differentiated frame is characterized by the fact that the singleton sets are closed and $\{w\}=\lim \mathfrak{U}_{w}$. Now $\{w\} \otimes-\{w\}=p \otimes \neg p\left[\lim \mathfrak{U}_{w}\right]=(\stackrel{\ddagger}{\ddagger})$ $\lim p \otimes \neg p\left[\mathfrak{U}_{w}\right] \subseteq \cup p \otimes \neg p=-\Delta$. Thus, even though the differentiated frames do not contain singletons, we can safely add them as values. Moreover, once we have added the singletons we can forget all other sets, since the singletons are decisive and consequently describability in $\mathcal{G}$ is reduced to describability in $\left(\mathcal{G}_{\sharp}\right)^{\sharp}$. Such reasoning, however, is conditional on the validity of the equation ( $\$)$ for the limits. Such equations for limits are not always true. But we can list a few easy results. First, if $\Delta$ is an upward going family of 
sets and $P$ is composed from variables with $\vee, \wedge, \exists$ then $\lim P[\Delta]=P[\lim \Delta]$. Moreover, if $\mathcal{G}$ is a continuous frame we can relax this considerably.

Lemma 35 Suppose that all projections $[\mathfrak{M}]_{i}$ are either positive or negative and that $\Delta$ is an up- or downgoing family of sets in a frame. Then $\mathfrak{M}[\lim \Delta]=\lim \mathfrak{M}[\Delta] \subseteq \cup \mathfrak{M}$.

Let us now agree to say that a family $\Delta$ of sets is smooth if it is a down- or upward going family such that for all positive or negative formulae $P \lim P[\Delta]=P[\lim \Delta]$. The above lemma states that in every continuous frame a closed set is the intersection of some smooth family. The same obviously holds for rough Kripke-frames; moreover, it holds for any finite union of continuous frames or rough Kripke-frames (whence the name approximation frames). We now give examples of the application of the technique. If $\mathfrak{S}$ is strongly positive and free of modalities and $\mathfrak{N}$ negative, then the atomic sets are decisive. Hence, $\mathcal{G} \vDash \alpha^{\dagger}=\bigcup \mathfrak{S} \otimes \mathfrak{N}$ for the natural translation $\alpha$ if only every singleton is a limit of a smooth family. The specific advantage is that choosing singletons as valuations makes the meaning of $\mathfrak{S} \otimes \mathfrak{N}$ perfectly clear. For example, by pretending the value of $p$ to be $\left\{w_{0}\right\}$ we can see that $p \otimes \square \diamond \square \neg p$ corresponds to $\left(\forall u \triangleright w_{0}\right)(\exists v \triangleright u)(\forall y \triangleright v)\left(y \neq w_{0}\right)$. For arbitrary square spones the cones, i.e. sets of type $\left\{s: w \triangleleft^{\kappa} s\right\}$ are decisive. Thus Sahlqvist's Theorem is proved by observing that the cones are smooth limits.

\section{References}

[Benthem, 1983] Johan van Benthem. Modal and Classical Logic. Bibliopolis, 1983.

[Benthem, 1984] Johan van Benthem. Correspondence theory. In Dov M. Gabbay and Franz Guenthner, editors, Handbook of Philosophical Logic, volume 2, pages 167247. Reidel, 1984.

[Chagrova, 1991] Lilia A. Chagrova. Undecidable problems in correspondency theory. The Journal of Symbolic Logic, 56:1261-1272, 1991.

[Fine, 1974] Kit Fine. Logics containing K4, Part I. The Journal of Symbolic Logic, 39:229-237, 1974.

[Fine, 1975] Kit Fine. Some connections between elementary and modal logic. In Stig Kanger, editor, Proceedings of the Third Scandinavian Logic Symposium, pages 15-31. North-Holland, 1975. 
[Fine, 1985] Kit Fine. Logics containing K4, Part II. The Journal of Symbolic Logic, 50:619-651, 1985.

[Goldblatt, 1976] Robert I. Goldblatt. Metamathematics of modal logics, Part I. Reports on Mathematical Logic, 6:41-78, 1976.

[Goldblatt, 1989] Robert I. Goldblatt. Varieties of complex algebras. Annals of Pure and Applied Logic, 38:173-241, 1989.

[Goldblatt, 1991] Robert I. Goldblatt. The McKinsey axiom is not canonical. The Journal of Symbolic Logic, 56:554 - 562, 1991.

[Hughes and Cresswell, 1984] George E. Hughes and Max J. Cresswell. A Companion to Modal Logic. Methuen, London, 1984.

[Jónsson and Tarski, 1951] Bjarni Jónsson and Alfred Tarski. Boolean algebras with operators. American Journal of Mathematics, 73:891-939, 1951.

[Kracht, 1991] Marcus Kracht. Internal Definability and Completeness in Modal Logic. PhD thesis, FU Berlin, 1991.

[Rautenberg, 1979] Wolfgang Rautenberg. Klassische und nichtklassische Aussagenlogik. Vieweg, Braunschweig/Wiesbaden, 1979.

[Sambin and Vaccaro, 1988] Giovanni Sambin and Virginia Vaccaro. Topology and duality in modal logic. Annals of Pure and Applied Logic, 37:249-296, 1988.

[Sambin and Vaccaro, 1989] Giovanni Sambin and Virginia Vaccaro. A new proof of Sahlqvist's theorem on modal definability and completeness. The Journal of Symbolic Logic, 54:992-999, 1989.

[Zakharyaschev, 1987] Michael Zakharyaschev. Syntax and semantics of modal logics containing S4. Algebra i Logika, 27:659-689, 1987. 